\title{
Rod Photoresponse Kinetics Limit Temporal Contrast Sensitivity in Mesopic Vision
}

\author{
Yumiko Umino, ${ }^{1}$ Ying Guo, ${ }^{1}$ Ching-Kang Chen, ${ }^{2}$ Rose Pasquale, ${ }^{1}$ and Eduardo Solessio ${ }^{1}$ \\ ${ }^{1}$ Center for Vision Research, Department of Ophthalmology, SUNY Upstate Medical University, Syracuse, New York 13210, and ${ }^{2}$ Departments of \\ Ophthalmology, Neuroscience, and Biochemistry and Molecular Biology, Baylor College of Medicine, Houston, Texas 77030
}

The mammalian visual system operates over an extended range of ambient light levels by switching between rod and cone photoreceptors. Rod-driven vision is sluggish, highly sensitive, and operates in dim or scotopic lights, whereas cone-driven vision is brisk, less sensitive, and operates in bright or photopic lights. At intermediate or mesopic lights, vision transitions seamlessly from rod-driven to cone-driven, despite the profound differences in rod and cone response dynamics. The neural mechanisms underlying such a smooth handoff are not understood. Using an operant behavior assay, electrophysiological recordings, and mathematical modeling we examined the neural underpinnings of the mesopic visual transition in mice of either sex. We found that rods, but not cones, drive visual sensitivity to temporal light variations over much of the mesopic range. Surprisingly, speeding up rod photoresponse recovery kinetics in transgenic mice improved visual sensitivity to slow temporal variations, in the range where perceptual sensitivity is governed by Weber's law of sensation. In contrast, physiological processes acting downstream from phototransduction limit sensitivity to high frequencies and temporal resolution. We traced the paradoxical control of visual temporal sensitivity to rod photoresponses themselves. A scenario emerges where perceptual sensitivity is limited by: (1) the kinetics of neural processes acting downstream from phototransduction in scotopic lights, (2) rod response kinetics in mesopic lights, and (3) cone response kinetics as light levels rise into the photopic range.

Key words: ERG; mesopic vision; mouse; operant behavior; temporal resolution; Weber adaptation

Significance Statement

Our ability to detect flickering lights is constrained by the dynamics of the slowest step in the visual pathway. Cone photoresponse kinetics limit visual temporal sensitivity in bright (photopic) lights, whereas mechanisms in the inner retina limit sensitivity in $\operatorname{dim}$ (scotopic) lights. The neural mechanisms underlying the transition between scotopic and photopic vision in mesopic lights, when both rods are cones are active, are unknown. This study provides a missing link in this mechanism by establishing that rod photoresponse kinetics limit temporal sensitivity during the mesopic transition. Surprisingly, this range is where Weber's Law of Sensation governs temporal contrast sensitivity in mouse. Our results will help guide future studies of complex and dynamic interactions between rod-cone signals in the mesopic retina.

\section{Introduction}

Under bright ambient conditions, human cone-driven (or photopic) vision can detect flicker at higher temporal frequencies than rod-driven vision under dim ambient conditions (de Lange

\footnotetext{
Received June 3, 2018; revised Jan. 29, 2019; accepted Jan. 30, 2019.

Author contributions:Y.U., Y.G., and E.S. wrote the first draft of the paper; Y.U., Y.G., C.-K.C., R.P., and E.S. edited the paper; Y.U., Y.G., C.-K.C., R.P., and E.S. designed research; Y.U., Y.G., and R.P. performed research;Y.U., Y.G., and E.S. analyzed data; Y.U. and E.S. wrote the paper.

This work was supported by the National Institutes of Health Grant R01 EY026216, the Department of Ophthalmology, State University of New York Upstate Medical University, an unrestricted Grant from Research to Prevent Blindness, and the Lions of Central New York. We thank Drs. Gus Engbretson, Bart Farell, and Dale Hunter for their insightful comments on an earlier version of the paper.

The authors declare no competing financial interests.

Correspondence should be addressed to Eduardo Solessio at solessie@upstate.edu.

https://doi.org/10.1523/JNEUROSCI.1404-18.2019

Copyright $\odot 2019$ the authors
}

Dzn, 1954; Kelly, 1961; Hess and Nordby, 1986). Despite the differences in the intrinsic response properties of rod and cone photoreceptors (Lamb, 2013), as light levels rise, the visual system transitions seamlessly from scotopic to photopic vision by way of mechanisms that are not understood (Stockman and Sharpe, 2006; Zele and Cao, 2014). The transition occurs in the mesopic light range, where both rod and cone photoreceptors actively respond to light and their signals are relayed along converging postsynaptic circuitry (MacLeod, 1972; van den Berg and Spekreijse, 1977; Völgyi et al., 2004; Cao et al., 2010).

The neural mechanisms that control the sensitivity of the visual system to temporal variations in light [also known as temporal contrast sensitivity (TCS)] reside largely in the retina (Dowling, 1967; Donner et al., 1990; Purpura et al., 1990; Tranchina et al., 1991; Seiple et al., 1992; Dunn et al., 2006, 2007; 
Smith et al., 2008; Jackman et al., 2009; Jarsky et al., 2011; Oesch and Diamond, 2011; Ke et al., 2014). The photoreceptor frequency response has been suggested to shape TCS in photopic lights (Tyler, 1985; Watson, 1986; Sharpe et al., 1989; Graham and Hood, 1992; Rovamo et al., 1999; Jarvis et al., 2003). In support of this view, a close link has recently been established between cone response kinetics and foveal/peripheral differences in sensitivity to fast flicker (Sinha et al., 2017). Thus cone photoresponse kinetics likely limit TCS in photopic conditions.

In contrast, behavioral observations in mice suggest that rod photoresponse kinetics do not limit TCS in dim scotopic conditions (0.4 photo-isomerizations/rod/s; Umino et al. 2012). Under these conditions, rods act as photon counters and process(es) downstream of phototransduction respond to the early rising phase of the photoresponse (Robson and Frishman, 1995; Field and Rieke, 2002; Sampath et al., 2005; Dunn et al., 2006; Okawa et al., 2010; Stockman et al., 2010). Rod photoresponse kinetics also do not limit TCS at the top of the scotopic range (50 photoisomerizations/rod/s; Peinado Allina et al. 2017). At this level rods integrate the response to multiple photo-isomerizations and govern the response kinetics of postsynaptic neurons (Fortenbach et al., 2015).

Here, we show that the kinetics of rod recovery determine visual (behavioral) TCS in mesopic but not in scotopic lighting. To perform these studies, we combined a forced-choice operant behavior assay and electrophysiology in mouse. We have previously established that mouse vision matches fundamental properties of human TCS using the operant assay (Umino et al., 2018). We compared TCS in control (WT) and mice overexpressing R9AP in their rods (line R9AP95; Chen et al., 2010, 2012). The expression level of R9AP sets the cellular levels of the GAP complex (RGS9·G $\beta 5 \cdot R 9 A P$ ) as R9AP overexpression results in an increase in RGS9 and G $\beta 5$-L levels (Krispel et al., 2006). RGS9 is a GTPase activating protein responsible for the rapid inactivation of the phototransduction G-protein, transducin (He et al., 1998). Overexpression of R9AP in rods speeds up their photoresponse recovery rates (Krispel et al., 2006; Chen et al., 2010, 2012; Peinado Allina et al., 2017). Our data show that fast rod inactivation kinetics differentially improves contrast sensitivity to low temporal frequency flicker in the mesopic range, where mouse vision operates in the Weber adaptation regime (Umino et al., 2018). Our electrophysiological recordings and mathematical modeling provide new insights to the complexity of rod-cone dynamics at mesopic light levels (Walraven et al., 1990; Stockman and Sharpe, 2006; Zele and Cao, 2014).

\section{Materials and Methods}

\section{Mouse genotype and husbandry}

Animal strains. We used heterozygous mice of the R9AP95 mouse line (Chen et al., 2010, 2012). In the remainder of this manuscript we refer to the R9AP95 line as R9OE (R9AP overexpressing). To selectively analyze rod-mediated responses under mesopic conditions we eliminated conedriven responses in mesopic lights by breeding R9AP95 lines onto the "black" GNAT2 $2^{\text {cpfl3 }}$ background (Chang et al., 2006). GNAT2 ${ }^{\text {cpfl3 }}$ mice (referred to as G2 in the text) carry a spontaneous point mutation in the GNAT2 gene that reduces cone phototransduction efficacy (Chang et al., 2006; Nusinowitz et al., 2007). As a result, mice have normal rod responses and desensitized cone responses that can be detected with intense flashes. Our R9OE and G2 lines were bred over seven generations into the C57BL6J background. Cones in black G2 mice are unresponsive in mesopic lights, and can be used to study rod vision in mesopic lights (Nathan et al., 2006; Naarendorp et al., 2010; Brown et al., 2011; Wang et al., 2011).
Animals of either sex, 3-6 month of age, were tested with an operant behavioral assay and flicker ERG recordings. Mice were maintained on a $14 / 10 \mathrm{~h}$ light/dark cycle, and tested during the subjective day. All procedures in this study were approved by the SUNY Upstate Medical University Institutional Animal Care and Use Committee (IACUC \#297) and were conducted in accordance with the Guide for the Care and Use of Laboratory Animals (National Academy of Sciences, Washington, DC) and in compliance with the Association for Research in Vision and Ophthalmology Statement for the Use of Animals in Ophthalmic and Vision Research. All efforts were made to minimize animal suffering.

\section{Operant conditioning assay to determine TCS in mice}

In our forced-choice operant studies, mice are trained to detect and respond to flickering lights, using a control and conditioning system (Lafayette Instruments) as described previously (Umino et al., 2018). Briefly, each mouse is maintained on a food-restriction schedule sufficient to provide necessary motivation to learn and perform the behavioral task (food is restricted to achieve $80-85 \%$ of expected body weight) and trained to perform a forced-choice visual task: discriminate presence or absence of flicker in the overhead LED-based test light $(505 \mathrm{~nm}$ central emission). A brief (20 ms), high-frequency tone is presented to alert the mice that they can initiate a trial. Trials are initiated when the mouse visits the reward tray. Following trial initiation, the light stimulus is presented concurrent with a cueing tone, both of which terminate when the animal responds, or in the absence of a response, after $12 \mathrm{~s}$. A response is computed as a visit to the reward tray following a visit to the left (flicker) or right (non-flicker) nose-poke ports. Correct responses (hits and correct rejections) are rewarded with a small amount of commercially available Ensure Nutrition Drink $(\sim 5-10 \mu \mathrm{l})$ on a schedule that rewards $100 \%$ of the hits and $100 \%$ of the correct rejections.

We test daily the ability of mice to detect flickering from nonflickering lights. Animals are dark-adapted for 1-2 $\mathrm{h}$ before the experimental session. Each session consists of 400 trials following a "warm up" session of 300 corrective trials. The duration of each session is $\sim 2-3 \mathrm{~h}$. During the sessions mice are continuously exposed to a fixed adapting light; the flicker test is a sinusoidally varying full-field illumination superimposed on the steady adapting light. The amplitude of the sinusoid increases gradually over a $1 \mathrm{~s}$ interval to reduce the generation of spillover frequencies. The level of the non-flicker test light is matched to the adapting light.

To determine temporal contrast sensitivity in mice we first measured their psychometric functions. Psychometric functions (PFs) are plots of the discriminability index, $d^{\prime}$, in response to different contrast levels. For the estimation of the discriminability factor $d^{\prime}$, we applied the following (Green and Swets, 1988; Macmillan and Creelman, 2005):

$$
d^{\prime}=z(H i t)-z(F A)
$$

where $z(\cdot)$ represents the $z$-score of the probability of hits or false alarms at a given contrast. The (empirical) contrast threshold, which depends on the relative shift of the PF along the contrast axis, was arbitrarily defined as the contrast necessary to elicit a $d^{\prime}=1$, which by the theory of signal detection (TSD) produces $76 \%$ correct responses in alternative forcedchoice tasks and is bias free. TCS is the inverse of the threshold.

Full PFs were determined in single, daily sessions consisting of 400 trials, where the magnitude of the contrast applied at each trial was variable and selected randomly from an array of five to six possible Rayleigh (or Michelson) contrast values (separated by $5 \%$ contrast intervals). Stimulus variables defined by the experimenter are mean light intensity $\left(I_{\mathrm{o}}\right)$, and temporal frequency $\left(f_{t}\right)$. We applied linear regression to determine the empirical contrast threshold at the intersection of the regression line with $d^{\prime}=1$. To reduce the uncertainty associated with day-to-day variability we measured and averaged PFs repeatedly until the regression coefficient $R^{2}$ for the cumulative average was 0.70 or higher, a process that requires averaging two to three PFs.

\section{Determination of retinal irradiance in freely behaving mice}

Levels of retinal irradiance were determined as described previously (Umino et al., 2018). Briefly, corneal irradiance (in Watts/area) was mea- 
A

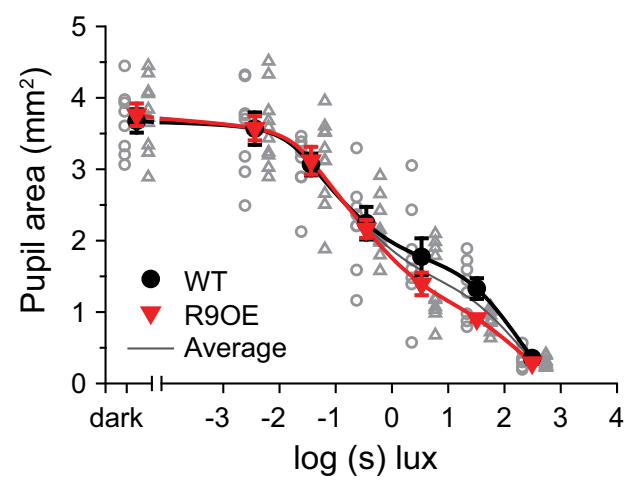

B

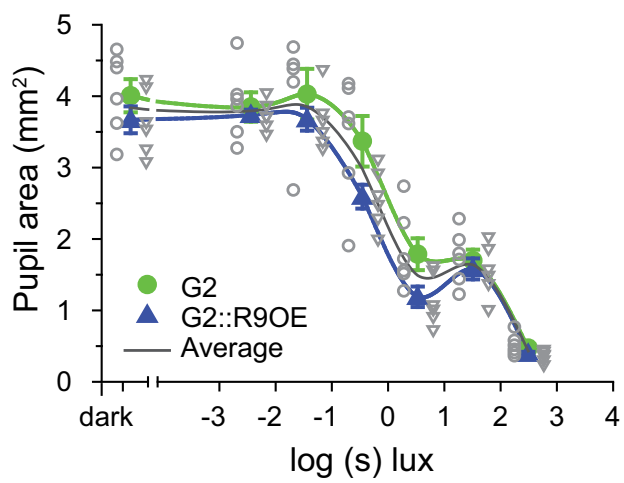

Figure 1. Pupil areas of freely behaving mice plotted as a function of scotopic illuminance. $\boldsymbol{A}$, Pupil areas plotted as a function of scotopic illuminance for wild-type $(n=8)$ and R90E $(n=10)$ mice; individual (gray circles and triangles, respectively) and average (WT, black; R90E, red) responses. Pupil areas of WT and R90E mice decreased gradually with illumination. There is no statistical difference in the pupil areas of WT and R90E mice [two-way RM ANOVA; Holm-Sidak post hoc test revealed no difference across genotypes $(p=0.35)$ or genotype $\times$ illuminance $(p=0.22)$ ]. Gray line is the response estimated by pooling the pupil areas of WT and R90E mice which was subsequently used for the calculation of their retinal irradiance levels. The pupil areas of R90E and WT match closely at low illuminance levels consistent with the notion that postsynaptic circuitry responds primarily to the rising phase of the rod photoresponse in dim lights (Robson and Frishman, 1995; Sampath et al., 2005), when rods act as single-photon counters. B, Pupil areas for G2 $(n=5)$ and G2::R90E mice $(n=6)$ individual (grey circles and triangles, respectively) and average (G2, green; $\mathrm{G} 2:: R 90 \mathrm{E}$, blue ) responses. Pupil areas of G2 and G2::R90E mice also decrease gradually with illumination, however a clear "pedestal" is observed in response to 0 -2 log ( $s$ ) lux, which we speculate reflects the contribution of different retinal mechanisms to the pupillary reflex (Lall et al., 2010; Keenan et al., 2016). We did not observe significant differences between genotype ( $p=0.12$ ) or genotype $\times$ irradiance ( $p=0.07$; two-way ANOVA). As a result, we pooled the pupil areas of G2 and G2::R90E mice (gray line) and used the pooled values to estimate retinal irradiance levels.

sured with an M370 Optometer (Graseby Optronics) and converted into scotopic illuminance units (s lux) using established formulas (Wyszecki and Stiles, 2000). Pupil areas of WT, R9OE, G2, and G2::R9OE mice behaving freely in the behavioral chamber were measured as a function of corneal illuminance using a custom-built portable device as described previously (Bushnell et al., 2016). Using pupil area and corneal illuminance information (Fig. 1) we applied the equations derived by Lyubarsky et al. (2004) to determine the levels of retinal irradiance at each experimental condition. Note that we express retinal irradiance in terms of photon flux at the retina $\left(\mathrm{ph} / \mathrm{s} / \mu \mathrm{m}^{2}\right)$ and not in the conventional form ( $\mathrm{R}^{\star} / \mathrm{rod} / \mathrm{s}$, where $\mathrm{R}^{*}$ are photo-isomerizations) because rod effective collecting area values are likely to change following prolonged bleaching $(2-3 \mathrm{~h})$ at the high irradiance levels used in our behavioral experiments (Lyubarsky et al., 2004).

Flicker ERG recordings

ERGs were recorded using the Espion E2 system and a ColorDome ganzfeld stimulator (Diagnosys) as described by Umino et al. (2012). Briefly, mice were dark-adapted overnight and prepared for recordings using dim red lights and infrared goggles. Mice were initially anesthetized by intraperitoneal injection of a ketamine/xylazine mixture (90 and $9 \mathrm{mg} /$ $\mathrm{kg}$, respectively). To maintain the animal under anesthesia over the $3 \mathrm{~h}$ experiments, booster applications at a lower dosage (30 and $3 \mathrm{mg} / \mathrm{kg}$ ) were applied approximately every $40 \mathrm{~min}$ via a wing infusion set placed subcutaneously in the flank of the animal. Pupils were dilated with $1 \%$ tropicamide and $2.5 \%$ phenylephrine hydrochloride. A drop of Gonak solution (2.5\%, Akorn) was applied on each cornea before the recordings. Recordings were performed with a conducting thread electrode placed in contact with the cornea and covered with a transparent contact lens to minimize cataract development. The reference electrode was placed in the mouth and a subcutaneous ground electrode positioned near the tail. Mouse body temperature was maintained at $37^{\circ} \mathrm{C}$ using an electric warming pad.

To isolate photoreceptor responses we injected intravitreally $1.5 \mu \mathrm{l}$ of a mixture containing $57 \mathrm{~mm}$ of L-(+)-2-amino-4-phosphonobutyric acid (L-AP4; catalog \#0103, Tocris Bioscience) and $86 \mathrm{~mm}$ of 2,3 cispiperidine dicarboxylic acid (PDA; ab120038, Abcam) in saline solution as described by Shirato et al. (2008) and Saszik et al. (2002). Briefly, the intravitreal injection was performed under a dissecting microscope and IR illumination. A 30 gauge needle was used to punch a hole just behind the limbus. An alumina glass pipette with a tip diameter of $25 \mu \mathrm{m}$ was inserted through the hole and $1.5 \mu \mathrm{l}$ mixture of the L-AP4 and PDA mixture was injected with a Hamilton microsyringe. We assume a mouse vitreal volume of $\sim 20 \mu \mathrm{l}$, resulting in estimated vitreal concentrations of 4 and $6 \mathrm{~mm}$ for L-AP4 and PDA, respectively. At the end of each experiment we tested for the appearance of the b-wave or the "positive intrusion" in the response to a brief flash superimposed on background illumination as described by Shirato et al. (2008). Presence of a b-wave or positive intrusion indicated washout (or bad injection) of the blocking mixture and the experiment was voided.

Flicker ERGs were evoked by sinusoidally modulated monochromatic light stimulus $(530 \mathrm{~nm})$ at various levels of mean retinal illuminance (40, $80,200,400,800,1600,3200$, and 6400 photons $\left./ \mathrm{s} / \mu \mathrm{m}^{2}\right)$, contrast $(75 \%)$, and temporal frequencies $(3,6$, and $12 \mathrm{~Hz})$. After electrode placement the animals were light-adapted for $1 \mathrm{~h}$ at a background level delivering $\sim 40$ photons $/ \mathrm{s} / \mu \mathrm{m}^{2}$ at the retina. The goal of the prolonged light adaptation period was to replicate the extended light adaptation conditions that the animals experience during the behavioral studies. Following the adaptation period, the background luminance was increased to a new level every $12 \mathrm{~min}$. The flicker stimulation sequence started $3 \mathrm{~min}$ after the transition to the new background level. Each sequence started with the presentation of flicker with $0 \%$ contrast to determine noise levels, followed by flicker stimulation of 3,6 , and $12 \mathrm{~Hz}$, each at $75 \%$ contrast. The sequence ended with a brief saturating flash from a Xenon lamp delivering $1778 \mathrm{~cd}$ $\mathrm{s} / \mathrm{m}^{2}$ to determine the fraction of dark current suppression at each background. Each flicker trial was $4 \mathrm{~s}$ in duration for $3 \mathrm{~Hz}$ flicker and $2 \mathrm{~s}$ for 6 and $12 \mathrm{~Hz}$. The response is the average of 30 trials. ERG responses were analyzed by fast Fourier transformation using MATLAB software (MathWorks) and the magnitude of the fundamental plotted as a function of retinal irradiance. The initial transient of the flicker response (5s) was not recorded.

Conversion from luminance to rate of rhodopsin excitation was performed by assuming that $1(\mathrm{scot}) \mathrm{cd} / \mathrm{m}^{2}$ generates $800 \mathrm{R} * / \mathrm{rod} / \mathrm{s}$ and the pupil area was $4 \mathrm{~mm}^{2}$ (Umino et al., 2012). As noted above in reference to the behavioral studies, rod effective collecting area values are likely to change following prolonged bleaching at the high irradiance levels used in our ERG experiments (Lyubarsky et al., 2004). Therefore, we express retinal irradiance for the ERGs in terms of photon flux at the retina $\left(\mathrm{ph} / \mathrm{s} / \mu \mathrm{m}^{2}\right)$ and not in the conventional form $\left(\mathrm{R}^{\star} / \mathrm{rod} / \mathrm{s}\right.$, where $\mathrm{R}^{\star}$ are photo-isomerizations). To convert rhodopsin excitation rates into retinal irradiance levels we divided the conversion factor by the end-on collecting area of mouse rods which is estimated at $\sim 0.87 \mu \mathrm{m}^{2}$ (Lyubarsky et al., 2004; Naarendorp et al., 2010). For practical purposes we assigned it a value of $1 \mu \mathrm{m}^{2}$. 


\section{Experimental design and statistical analysis}

For the temporal contrast sensitivity functions involving both WT and R9OE mice, a two-way repeated-measures (RM) ANOVA was used with the nominal factors being genotype and frequency. Holm-Sidak's procedure for pairwise multiple comparisons was performed to test the hypothesis that mean measurements obtained from R9OE mice were not different from WT mice. A similar analysis was applied to comparisons between R9OE::G2 and G2 mice. When necessary, logarithmic transformations of contrast sensitivity data were performed before statistical analysis to fulfill normality and equal variance requirements for the ANOVA. In the case of the contrast sensitivity versus retinal irradiance functions (behavior) or magnitude versus retinal irradiance (ERG data), two-way RM ANOVA was performed independently at each temporal frequency. Data analysis was performed with SigmaStat software (Systat Software). Plots display mean \pm SEM. Numbers of mice and $p$ values are indicated in the text or figure legends.

\section{Model equations}

Linear stage. For a sinusoidally modulated flicker input given by the following:

$$
I(t)=I_{o}[1+C \sin (w t)],
$$

where $C$ is the modulation contrast, Io the mean irradiance, $w=2 \pi f_{o}$, is the flicker in radians per second, and $f_{o}$ is the flicker frequency in Hertz. The response is given by the convolution of the flicker input and the impulse response of the "transfer function" as follows:

$$
x(t)=I(t) * h(t) .
$$

The impulse response of a first-order integrating stage is defined as follows:

$$
h(t)=h_{o} e^{-t / \tau}=h_{o} e^{-a t},
$$

where ho is the amplitude of the "impulse" response, $\tau$ is the time constant of the exponential response, and the rate of the reaction is $a=\tau^{-1}$. The area under the exponential function $(A)$ is given by the product of the time constant by the amplitude such that: $A=\tau h_{o}$

Solution of Equation 3 results in the following:

$$
x(t)=\frac{h_{o} I_{o}}{a}\left(1-e^{-a t}\right)+\frac{h_{o} I_{o} C}{a^{2}+w^{2}}\left[\sqrt{a^{2}+w^{2}} \sin (w t+\varphi)+w e^{-a t}\right],
$$

where

$$
\varphi=\tan ^{-1}\left(-\frac{\mathrm{w}}{\mathrm{a}}\right)
$$

is the phase of the response. After a sufficiently long time:

$$
t \gg 1 / a=\tau,
$$

the initial transient of the response decays and Equation 5 simplifies to the steady response of the linear model:

$$
x_{s s}(t)=\frac{h_{o} I_{o}}{a}+\frac{h_{o} I_{o} C}{\sqrt{a^{2}+w^{2}}} \sin (w t+\varphi) .
$$

Linear-nonlinear model. Assuming that the nonlinearity is defined by the Hill equation, the output is given by the following:

$$
y(t)=K \frac{h(t) * I(t)}{h(t) * I(t)+E C_{50}},
$$

where $K$ is a calibration constant and $E C_{50}$ indicates the irradiance level that produces $50 \%$ of the maximal output. Substituting terms in Equation 9 we obtain the following:

$$
y(t)=K \frac{h_{o} e^{-a t} * I_{o}[1+C \sin (w t)]}{h_{o} e^{-a t} * I_{o}[1+C \sin (w t)]+E C_{50}} .
$$

Which can be solved for the steady state $(t \gg 1 / a=\tau$, condition 7$)$ and expressed as follows:

$$
y_{s s}(t)=K \frac{\frac{h_{o} I_{o}}{a}+\frac{h_{o} I_{o} C}{\sqrt{a^{2}+w^{2}}} \sin (w t+\varphi)}{\frac{h_{o} I_{o}}{a}+\frac{h_{o} I_{o} C}{\sqrt{a^{2}+w^{2}}} \sin (w t+\varphi)+E C_{50}} .
$$

To simplify the notation we define the inverse of the exponential integration area as:

$$
\gamma=\frac{a}{h_{o}}=\frac{1}{\tau h_{o}}
$$

and the attenuation factor of the linear filter as:

$$
\beta=\frac{a C}{\sqrt{a^{2}+w^{2}}}
$$

Where $\tau$ has units of time (seconds), the factor $h_{o}$ has units of seconds ${ }^{-1}$, and both $I_{o}$ and $E C_{50}$ have units of irradiance $\left(\mathrm{ph} / \mathrm{s} / \mu \mathrm{m}^{2}\right)$.

Hence we rewrite Equation 11 as follows:

$$
y_{s s}(t)=K \frac{I_{o}+\beta I_{o} \sin (w t+\varphi)}{I_{o}+\beta I_{o} \sin (w t+\varphi)+\gamma E C_{50}} .
$$

From Equation 14, we readily derive the magnitude of the response in steady state as follows:

$$
\Delta y_{s s}=\frac{K \gamma \beta I_{0} E C_{50}}{\left(1-\beta^{2}\right) \gamma^{2} I_{0}^{2}+2 \gamma I_{0} E C_{50}+E C_{50}^{2}},
$$

and estimate the peak response as a function of the attenuation factor $\beta$ :

$$
\hat{\Delta}_{s s}=\frac{K}{2} \frac{\beta}{1+\sqrt{1-\beta^{2}}},
$$

at an irradiance value of the following:

$$
\left.I_{o}\right|_{\text {Max }}=\frac{\gamma E C_{50}}{\sqrt{1-\beta^{2}}} .
$$

We note that when the attenuation factor $\beta^{2} \ll 1$, then:

$$
\begin{gathered}
\hat{\Delta} y_{s s}=\frac{K}{4} \frac{a C}{w}=\frac{K}{4} \frac{C}{\tau w}, \\
\left.I_{o}\right|_{\text {Max }}=\frac{E C_{50}}{\tau h_{o}} .
\end{gathered}
$$

In other words, the model predicts that both the peak of the response $\hat{\Delta} y_{s,}$ and the irradiance at the peak $I_{o} \mid \max$ are inversely related to the value of the time constant $(\tau)$. In addition the peak response $\hat{\Delta} y_{s s}$ is also inversely related to the frequency of the flicker stimulation $w$, which is consistent with results shown in Figure 4.

\section{Results}

Rod inactivation kinetics limit temporal contrast sensitivity to low temporal frequencies

We applied an operant behavior assay to determine TCS functions (TCSFs) in mice using a yes-no (one-forced choice) paradigm (see Materials and Methods for details). TCSFs are plots of TCS measured at multiple background illumination levels and temporal frequencies. We have previously shown that TCSFs in mice share many key features with TCSFs in humans (Umino et al., 2018). Here we test the hypothesis that rod inactivation kinetics shapes TCSFs in mice. We compared TCSFs in control (WT) and R9AP95 mice overexpressing R9AP in rods (in the text we 
A

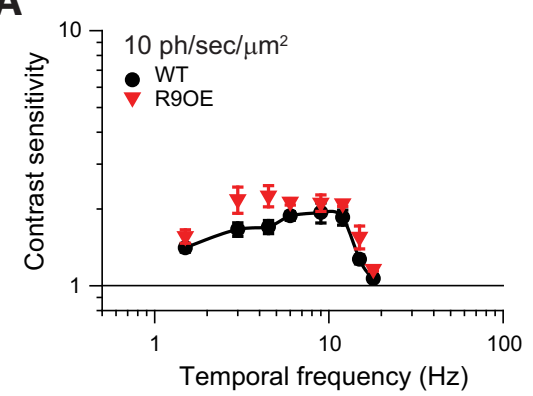

B

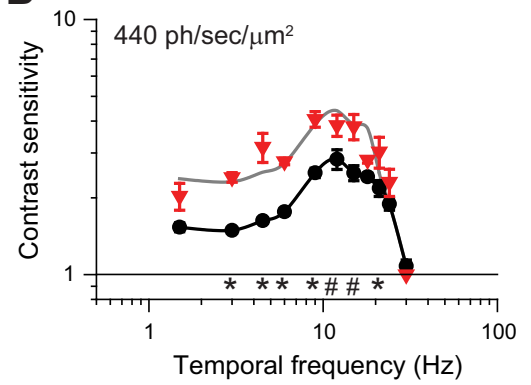

C

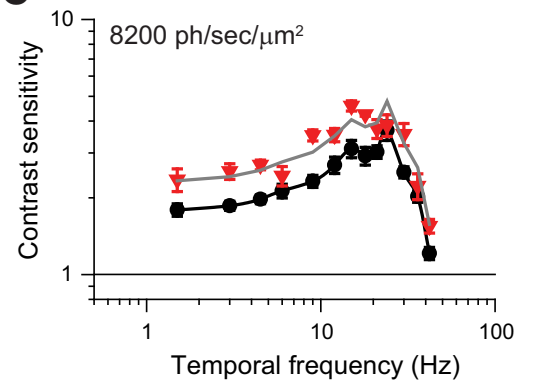

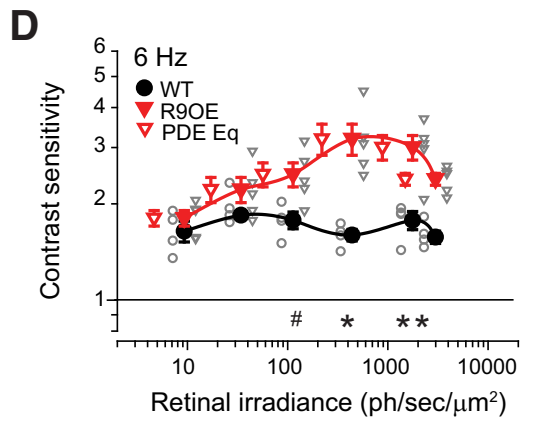

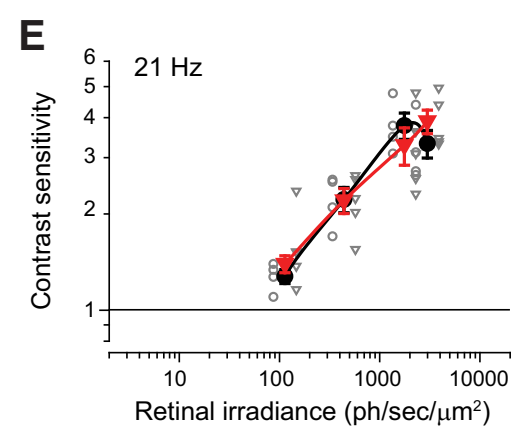

Figure 2. Rod response kinetics limit TCS to low temporal frequencies when mouse vision operates in Weber's adaptation regime. $\boldsymbol{A}-\boldsymbol{C}$, Temporal contrast sensitivity functions of WT (black circles) and R90E (red triangles) mice measured in: $(\boldsymbol{A}) \operatorname{dim}\left(10 \mathrm{ph} / \mathrm{s} / \mu \mathrm{m}^{2}\right.$ at the retina, $\left.n=4\right),(\boldsymbol{B})$ intermediate (400 photons $\left./ \mathrm{s} / \mu \mathrm{m}^{2}, n=4\right)$, and $(\boldsymbol{C})$ bright $\left(8200 \mathrm{ph} / \mathrm{s} / \mu \mathrm{m}^{2}, n=7-12\right) \mathrm{background}$ lights. Gray lines represent control values scaled by a factor of 1.5 (frequencies $<15 \mathrm{~Hz}$ ) in $(\boldsymbol{B})$ and 1.2 (all frequencies) in $\boldsymbol{C} . \boldsymbol{D}, \boldsymbol{E}$, Temporal contrast sensitivity of WT (black circles; $n=4$ ) and R90E (red triangles; $n=5$ ) plotted as a function of retinal irradiance, in response to $6 \mathrm{~Hz}(\boldsymbol{D})$ or $21 \mathrm{~Hz}(\boldsymbol{E})$ flicker stimulation. Also shown are individual data (open symbols in gray, displaced laterally for illustration purposes) and TCS of R90E mice after accounting for equivalent PDE activation (PDE-Eq; $\boldsymbol{D}$, red open triangles). Statistical analysis: two-way RM ANOVA, frequency $\times$ genotype interactions, $\# p<0.05,{ }^{*} p<0.001$, all other interactions $p>0.05$. See text for details. Symbols represent mean \pm SEM. Retinal irradiance estimates were performed as detailed in Materials and Methods.

refer to this line of mice as R9OE) measured with the operant behavior assay. Overexpression of R9AP in rods speeds up their photoresponse recovery rates (Krispel et al., 2006; Chen et al., 2010; Peinado Allina et al., 2017) and increases the amplitude of their responses to flicker stimulation (Fortenbach et al., 2015). We found that much like WT mice, TCS functions of R9OE mice exhibit the classic low to bandpass transformation as background light levels rise (Fig. 2), as observed in humans, however, with important light-and frequency-dependent differences relative to WT mice.

In $\operatorname{dim}$ lights $\left(10 \mathrm{ph} / \mathrm{s} / \mu \mathrm{m}^{2}\right.$ at the retina; see Materials and Methods for estimation of irradiance values), R9OE and WT mice had similar low-pass TCS functions (Fig. 2A). A small increase in average sensitivities to 3 and $4.5 \mathrm{~Hz}$ in R9OE mice was not statistically significant (no significant interaction between genotype and frequency $(p=0.6)$ and no significant difference in genotype ( $p=0.1$ ); two-way RM ANOVA). At these dim light levels rods integrate single-photon responses and begin to activate their adaptation mechanisms (Dunn et al., 2006). These results are in line with those reported previously by Peinado Allina et al. (2017) using a wheel-running visual behavioral apparatus.

At intermediate light levels $\left(440 \mathrm{ph} / \mathrm{s} / \mu \mathrm{m}^{2}\right.$ at the retina), R9OE mice exhibited a frequency-dependent increase in sensitivity relative to WT mice (Fig. 2B). TCSFs of both R9OE and WT mice exhibited the trademark bandpass shape as seen in human (de Lange Dzn, 1958; Kelly, 1961; Watson, 1986; Umino et al., 2018), however, R9OE mice were more sensitive than WT mice to flicker frequencies $<15 \mathrm{~Hz}$, although their sensitivity to frequencies $>15 \mathrm{~Hz}$ remained normal (significant interaction between genotype and frequency; $p=0.002$, power $=0.9$; two-way RM ANOVA).
At bright light levels near the upper end of the mesopic range $\left(8200 \mathrm{ph} / \mathrm{s} / \mu \mathrm{m}^{2}\right.$ at the retina), the sensitivity of R9OE mice was higher than that of WT mice (Fig. 2C) at all stimulus frequencies (no significant interaction between genotype and frequency; $p=$ 0.27 ; but significant difference in genotype; $p=0.027$; two-way RM ANOVA). Scaling the TCSFs of WT mice by a constant factor (in this case 1.25) closely fit the TCS function of R9OE mice. Together, comparisons of the TCS functions (Fig. $2 A-C$ ) suggest that TCS is limited by R9AP expression in rods in a complex way that depends both on temporal frequency and light levels.

\section{Photoreceptor response kinetics control TCS during Weber's adaptation}

To better understand how light adaptation properties differ in R9OE and WT mice we measured TCS to low $(6 \mathrm{~Hz})$ and high $(21$ $\mathrm{Hz}$ ) flicker stimulation using small (0.6 log unit) increments in mean irradiance. TCS of WT mice to $6 \mathrm{~Hz}$ flicker remained constant over a $3.5 \log$ range in retinal illumination $(10-3000 \mathrm{ph} / \mathrm{s} /$ $\mu \mathrm{m}^{2}$ at the retina), consistent with Weber's law (Fig. $2 D$; no significant differences were observed, see statistics at the end of the paragraph). On the other hand, TCS of R9OE mice followed a non-monotonic relation with background light levels: TCS was normal at low light levels $\left(<110 \mathrm{ph} / \mathrm{s} / \mu \mathrm{m}^{2}\right.$ at the retina), but grew gradually with higher light intensities, reaching maximal sensitivities for irradiance values ranging from 440 to $1700 \mathrm{ph} / \mathrm{s} /$ $\mu \mathrm{m}^{2}$ (Fig. $2 D$; significant interaction between genotype and frequency; $p<0.001$ and power $=0.9$; comparisons of WT responses within irradiance were not significantly different; $p>$ 0.35; two-way RM ANOVA). TCS of R9OE mice decreased with irradiance levels $>1700 \mathrm{ph} / \mathrm{s} / \mu \mathrm{m}^{2}$. 


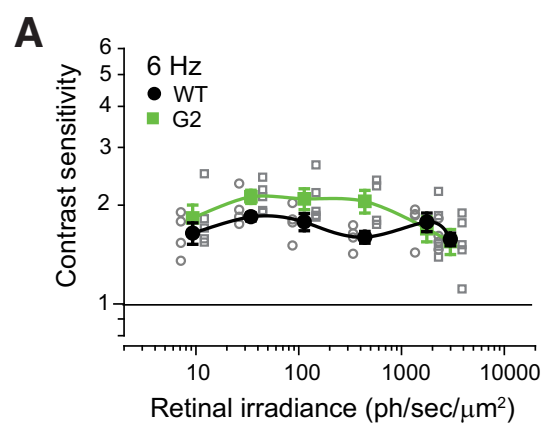

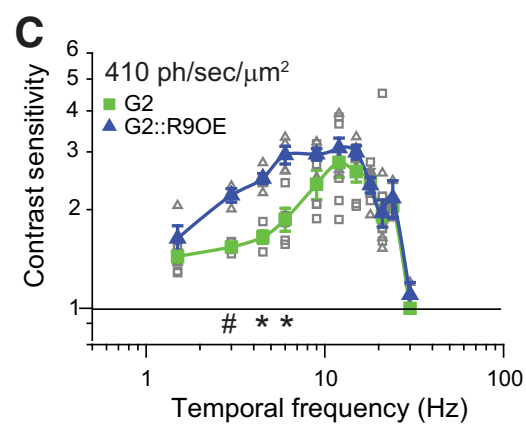

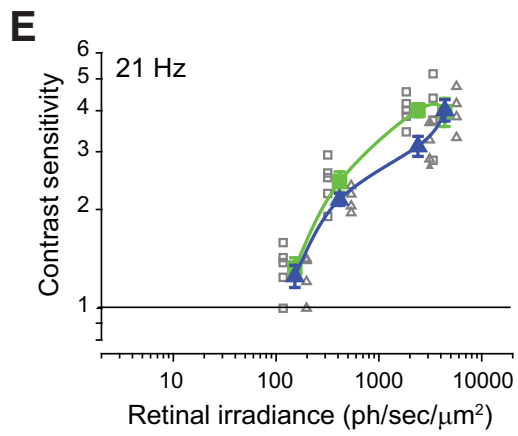

Figure 3. High TCS in R90E mice does not arise from rod-cone interactions. $A, B$, Temporal contrast sensitivity of WT (black circles, same as in Fig. $2 A, B ; n=4$ ) and G2 (green squares; $n=5$ ) mice plotted as a function of retinal irradiance in response to $6 \mathrm{~Hz}(\boldsymbol{A})$ or $21 \mathrm{~Hz}(\boldsymbol{B})$ flicker stimulation. Also shown is TCS of individual data (open symbols in gray, displaced laterally for illustration purposes). Statistical analysis: two-way RM ANOVA, Holm-Sidak method to test frequency $\times$ genotype interactions, $\# p<0.05,{ }^{*} p<0.001$, all other interactions $p>0.05$. See text for details. Error bars indicate SEM. Note that in this study, WT and G2 mice received the same illuminance levels at the pupil. Although the pupil areas differed slightly (see Materials and Methods), the respective differences in retinal irradiance levels in WT and $\mathrm{G} 2$ mice were relatively small ( $<0.16 \mathrm{log}$ units) and did not impact this analysis. $C$, Temporal contrast sensitivity functions of G2 (green squares; $n=$ 6) and G2::R90E (blue triangles; $n=5$ ) mice measured in intermediate background lights ( $410 \mathrm{ph} / \mathrm{s} / \mu \mathrm{m}^{2}$ at the retina). Open gray symbols are individual responses. $\boldsymbol{D}, \boldsymbol{E}$, Temporal contrast sensitivity of G2 (green squares; same data as in $\boldsymbol{A}$ and $\boldsymbol{B}$ ) and G2::R90E (blue triangles; $n=4$ ) plotted as a function of retinal irradiance in response to $6 \mathrm{~Hz}(\boldsymbol{D})$ or $21 \mathrm{~Hz}(\boldsymbol{E})$ flicker stimulation. Also shown is TCS of G2::R90E mice after accounting for equivalent PDE activation (PDE-Eq; $\boldsymbol{D}$, open blue triangles) and individual data (open symbols in gray). Statistical analysis: same as in $\boldsymbol{A}$ and $\boldsymbol{B}$.

Unlike the responses to $6 \mathrm{~Hz}$ flicker, TCS to $21 \mathrm{~Hz}$ flicker did not adapt to background light levels and TCS to $21 \mathrm{~Hz}$ flicker is independent of the level of R9AP expression (Fig. 2E). Responses to $21 \mathrm{~Hz}$ were first observed in background lights delivering 110 $\mathrm{ph} / \mathrm{s} / \mu \mathrm{m}^{2}$ at the retina. Thereafter, TCS of both WT and R9OE mice rose gradually with irradiance [Fig. 2E; nonsignificant difference in genotype $(p=0.43)$, or interaction between genotype and frequency ( $p=0.18)$; two-way RM ANOVA]. Activation at high irradiance levels and lack of adaptation in response to high temporal frequencies (e.g., $21 \mathrm{~Hz}$ flicker) is also a feature of human TCS (Shapley and Enroth-Cugell, 1984).

\section{High TCS in R9OE mice does not arise from differential rod PDE activation}

As a result of their faster response kinetics, rods with higher levels of transducin GAP have shorter integration times than control rods (Krispel et al., 2006; Chen et al., 2010), leading to smaller steady-state responses to steps of light and a relative shift of their response-intensity relationship to the right by $0.3 \mathrm{log}$ units (twofold; Fortenbach et al., 2015). Hence, at equal steady irradiance levels, WT and R9OE rod photoreceptors rods are differentially activated via phosphodiesterase (PDE)- and calcium-dependent adaptation mechanisms (Yau and Nakatani, 1985; Matthews et al., 1988; Nikonov et al., 2000). To determine whether differential rod activation may explain the higher TCS to $6 \mathrm{~Hz}$ flicker that we observe in R9OE versus WT mice, we matched the levels of dark current suppression (steady rod activation) at each irradiance level as per Fortenbach et al. (2015). This was accomplished by shifting the TCS curve for R9OE mice to the left by 0.3 log units (Figs. 2D, red open triangles). We find that even with the leftward shift, TCS to $6 \mathrm{~Hz}$ flicker of R9OE mice remains significantly higher than that of WT mice. Indeed, our results suggest that TCS in R9OE mice is better explained by a vertical rather than a horizontal shift of the TCS of WT mice. Hence, we conclude that differential steady-state activation of R9OE rods cannot fully explain the increase in TCS to $6 \mathrm{~Hz}$.

\section{High TCS in R9OE mice does not arise from} rod-cone interactions

TCS is subject to phase differences in rod-rod (Stockman et al., 1991), and rod-cone signal interactions (MacLeod, 1972; van den Berg and Spekreijse, 1977; Zele and Cao, 2014). Given that fast inactivation kinetics of R9OE rods (Krispel et al., 2006; Chen et al., 2010) alter the phase of their responses to sinusoidal stimulation (see results in the next section), we examined whether abnormal rod-cone interactions underlie the changes in TCS of R9OE mice (Fig. 3). Our approach was to selectively isolate rodmediated vision in animals that have attenuated cone responses because of a missense mutation in the cone-specific transducin $\alpha$-subunit gene (Gnat2, "G2"; GNAT2 ${ }^{\text {cpfl3 }}$; Chang et al. (2006); Nusinowitz et al. (2007)). Behavioral and ERG controls performed in our lab confirm that G2 mice have no cone responses at the "mesopic" retinal irradiance levels used in this study (100$4000 \mathrm{ph} / \mathrm{s} / \mu \mathrm{m}^{2}$ ). At $6 \mathrm{~Hz}$, TCS of WT and G2 mice match closely at retinal irradiance levels ranging from 10 to $\sim 4000 \mathrm{ph} / \mathrm{s} / \mu \mathrm{m}^{2}$ (no interactions between genotype and illuminance, $p=0.38$; two-way RM ANOVA; Fig. 3A). TCS to $21 \mathrm{~Hz}$ did not differ in G2 and WT mice [Fig. $3 B$; no differences between genotype $(p=$ $0.54)$, or interactions between genotype and illumination $(p=$ 0.12); two-way RM ANOVA]. Altogether these results are consis- 
tent with the notion that rods and not cones drive TCS at retinal irradiance levels $<4000 \mathrm{ph} / \mathrm{s} / \mu \mathrm{m}^{2}$. Such an extended range of rod-driven behavior is unexpected given that the threshold for cone-driven responses to incremental flashes is in the order of $100 \mathrm{R} * / \mathrm{rod} / \mathrm{s}$ (Naarendorp et al., 2010). These results highlight the dynamic nature of rod-versus cone-driven sensitivities in the mesopic range.

Next we tested TCS in G2 mice with fast rod kinetics. We found that mice with fast rod responses and no (mesopic) cone responses (G2::R9OE double mutant mice) exhibited enhanced TCS to low frequencies relative to their G2 control counterparts (Fig. 3C). G2::R9OE and G2 mice have similar TCS at $6 \mathrm{~Hz}$ for retinal irradiance levels at or $<40 \mathrm{ph} / \mathrm{s} / \mu \mathrm{m}^{2}$. However, TCS of G2::R9OE mice was significantly higher than that of G2 mice at 6 $\mathrm{Hz}$ for retinal irradiance levels ranging from $\sim 100$ to $4000 \mathrm{ph} / \mathrm{s} /$ $\mu \mathrm{m}^{2}$ (genotype $\times$ intensity interactions, $p<0.001$; two-way $\mathrm{RM}$ ANOVA; individual interactions indicated in Fig. $3 D$ ). Indeed, TCS of G2::R9OE mice remained relatively constant at irradiance levels ranging from 150 to $2400 \mathrm{ph} / \mathrm{s} / \mu \mathrm{m}^{2}$ consistent with the notion of a shift in the Weber adaptation regime to higher TCS values $(\sim 3)$. A similar trend was observed with R9OE mice (Fig. $2 D$ ), although over a reduced irradiance range that extends from 440 to $1700 \mathrm{ph} / \mathrm{s} / \mu \mathrm{m}^{2}$. No significant differences in TCS to $21 \mathrm{~Hz}$ were detected in G2::R9OE and G2 mice (Fig. 3E; no differences between genotype, $p=0.15$, or genotype $\times$ intensity interactions, $p=0.21$; two-way RM ANOVA). Altogether, these results confirm that, for retinal irradiance levels ranging from $\sim 100$ to $4000 \mathrm{ph} / \mathrm{s} / \mu \mathrm{m}^{2}$, the increase in TCS to low frequencies in R9OE mice does not arise from rod-cone interactions.

\section{Pharmacologically-isolated ERG responses of WT and R9OE mice are consistent with behavioral sensitivity}

To understand how rod photoresponse kinetics limits TCS to low frequencies we assessed the responses of WT and R9OE rods electroretinographically to the same stimulus conditions applied in the operant behavior trials. We performed these measurements in vivo because: (1) photoreceptor kinetics are strongly dependent on the cellular environment (Azevedo and Rieke, 2011; Vinberg et al., 2014; Peinado Allina et al., 2017); and (2) conditions of our behavioral experiments require that we assess rod responses during prolonged exposure to background lights $(>1 \mathrm{~h})$, as well as in the presence of a working visual cycle. Thus, we examined the influence of accelerated transducin deactivation and background illumination on the frequency response of photoreceptors using in vivo flicker ERGs (see Materials and Methods for details). Photoreceptor contributions to the ERG were isolated pharmacologically with an intravitreal mixture containing glutamate receptor agonists and antagonists (Kondo and Sieving, 2001; Shirato et al., 2008). The isolated ERG reflects contributions of photoreceptors as well as a slow negative going slow PIII component (Shirato et al., 2008; see Discussion). Contrast level was set at 75\%, which is slightly higher than the TCS thresholds of WT mice determined with the behavioral assay. Lower contrast levels did not produce measureable responses to $6 \mathrm{~Hz}$ flicker in WT mice.

We tested the pharmacologically-isolated ERG responses of WT and R9OE mice to sinusoidal flicker stimulation $(3 \mathrm{~Hz})$ of constant contrast $(75 \%)$ presented at three different mean retinal irradiance levels (Fig. $4 A$ ). At low irradiance levels (40 ph/s/ $\mu \mathrm{m}^{2}$ ), the flicker ERG responses of R9OE and WT mice were small and overlapped closely, suggesting that R9AP-overexpression does not influence rod responses at dim irradiance levels. The amplitude of the responses grew differentially at intermediate irradiance levels $\left(800 \mathrm{ph} / \mathrm{s} / \mu \mathrm{m}^{2}\right)$ such that R9OE mice had markedly higher amplitudes and faster responses (as inferred from the relative phase advance of the waveforms) than WT mice. At high irradiance levels $\left(6400 \mathrm{ph} / \mathrm{s} / \mu \mathrm{m}^{2}\right)$ the amplitude of the responses decreased in both genotypes relative to that at intermediate irradiance levels; however, the response amplitudes remained higher in R9OE mice compared with WT mice. A similar trend but scaled down in amplitude was present in response to $6 \mathrm{~Hz}$ flicker (Fig. 4A, right).

To quantify the amplitude of the responses, we computed the Fast Fourier Transform of the response traces and plotted the magnitude of the fundamental frequency $(F o)$ as a function of mean retinal irradiance (Fig. $4 B$ ). In response to $3 \mathrm{~Hz}$ flicker the plots follow non-monotonic relations that peak slightly $>400$ $\mathrm{ph} / \mathrm{s} / \mu \mathrm{m}^{2}$ for WT and $800 \mathrm{ph} / \mathrm{s} / \mu \mathrm{m}^{2}$ for R9OE mice. Maximal response magnitudes of R9OE mice are significantly larger than those of WT mice (significant genotype $\times$ irradiance interaction; $p=0.005$ and power $=0.8$; two-way RM ANOVA; individual interaction levels are shown in Fig. $4 B$, middle). A similar response pattern was observed in response to $6 \mathrm{~Hz}$ flicker, where the non-monotonic relations again peak at $\sim 400$ and $800 \mathrm{ph} / \mathrm{s} / \mu \mathrm{m}^{2}$ for WT mice and R9OE mice, respectively, and the response magnitudes of R9OE mice are significantly larger than those of WT mice (significant genotype $\times$ irradiance interaction; $p<0.005$ and power $=0.8$; two-way RM ANOVA; individual interaction levels are shown in Fig. $4 B$, bottom). Interestingly, the isolated ERG responses of $\mathrm{R} 9 \mathrm{OE}$ mice to $6 \mathrm{~Hz}$ flicker peak at approximately the same retinal irradiance levels $\left(800 \mathrm{ph} / \mathrm{s} / \mu \mathrm{m}^{2}\right)$ as their behavioral contrast sensitivities (compare Fig. $2 D$ ), whereas the isolated ERG responses of WT mice to $6 \mathrm{~Hz}$ remain relatively flat, much the same as their corresponding behavioral responses. We further investigate the relation between ERG magnitudes and behavioral responses below.

We determined the phase of the responses by fitting the time course of the individual responses with sine wave functions. Plots of phase versus irradiance (Fig. 4C) show that the phase of WT responses to $3 \mathrm{~Hz}$ remained relatively constant across the range $\left(40-6400 \mathrm{ph} / \mathrm{s} / \mu \mathrm{m}^{2}\right)$, however, the phase of R9OE mice responses increased $\sim 0.4$ radians before leveling off at irradiance levels $>400 \mathrm{ph} / \mathrm{s} / \mu \mathrm{m}^{2}$ [statistical difference between genotypes ( $p=0.04)$ but no significant interactions between genotype and irradiance $(p=0.25)$; two-way RM ANOVA]. The phase advance in the response of $\mathrm{R} 9 \mathrm{OE}$ mice is consistent with their faster response kinetics (see next section), whereas the gradual increase in phase with low irradiance levels can be attributed to light adaptation mechanisms that speed up photoreceptor response kinetics (Tranchina et al., 1984; Smith et al., 2001). The phase of the responses to $6 \mathrm{~Hz}$ are relatively constant within the irradiance range of interest; however, the difference in phase is reduced to $0.1-0.2$ radians. We note that, in response to $6 \mathrm{~Hz}$, the fits to individual traces had $R^{2}<0.7$; hence, we averaged the response traces of all mice (Fig. $4 A$ ) which considerably improved the sine wave fits $\left(R^{2}>0.8\right)$. The pharmacologically-isolated flicker ERGs of WT and R9OE mice have the same magnitude and phase as the isolated ERGs of G2 and G2::R9OE mice suggesting that, within the stimuli range of interest, the isolated ERG signal is driven primarily by rods and not cones (Fig. 4D).

The horizontal separation between the peaks of the WT and R9OE ERG responses ( $0.3 \log$ units) agrees with the separation between the steady response curves of WT and R9OE rods (Fig. $4 B$, top) as recorded by Fortenbach et al. (2015). This suggests that the non-monotonic shape of the magnitude versus irradiance relations can be attributed to the compressive nonlinearity 
A
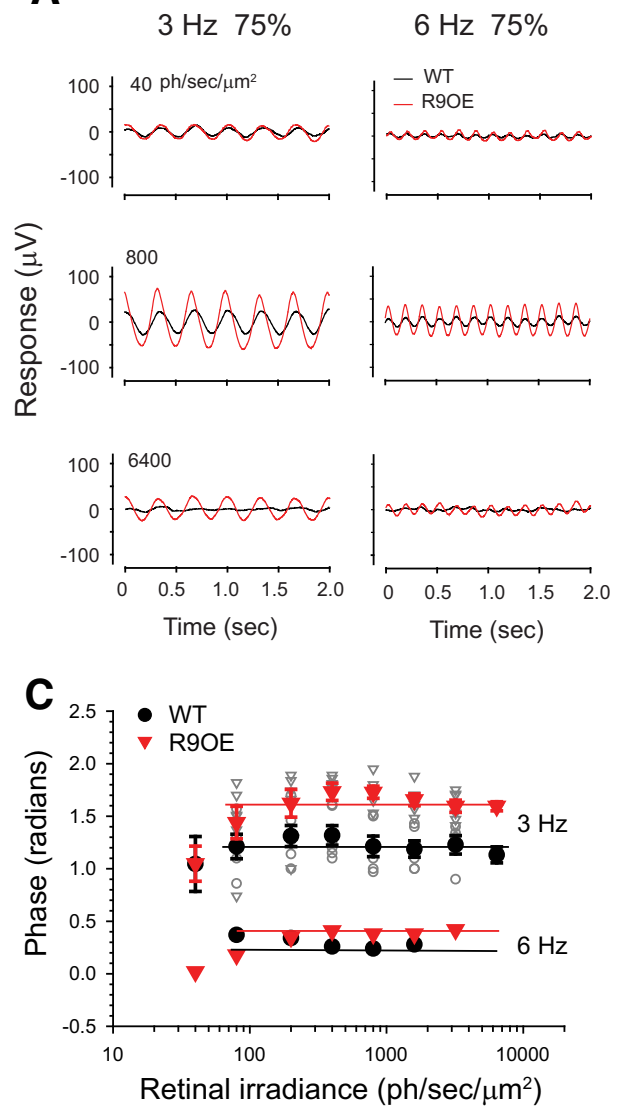

B
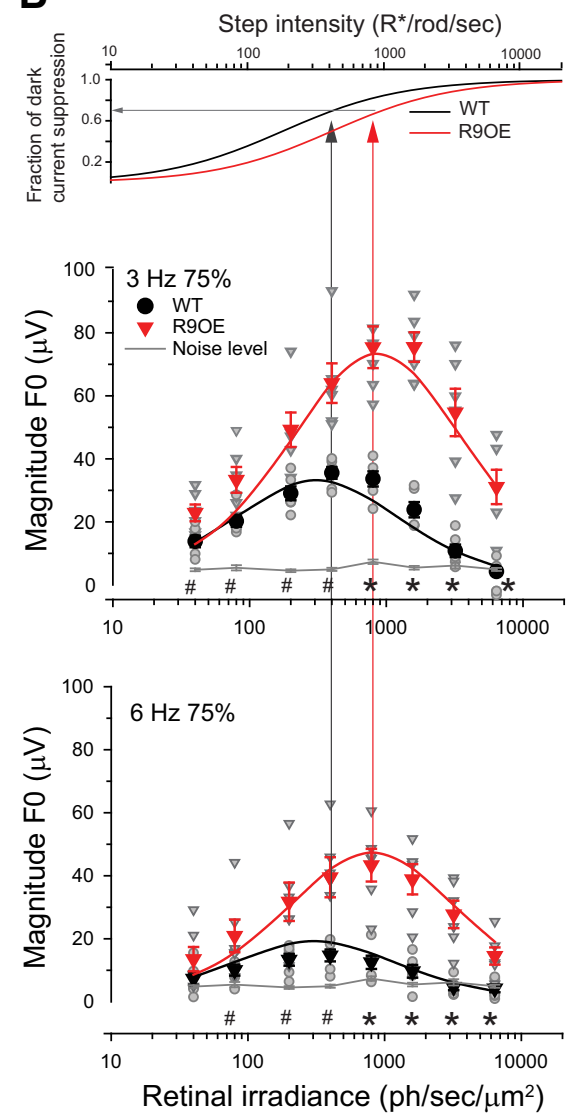

D
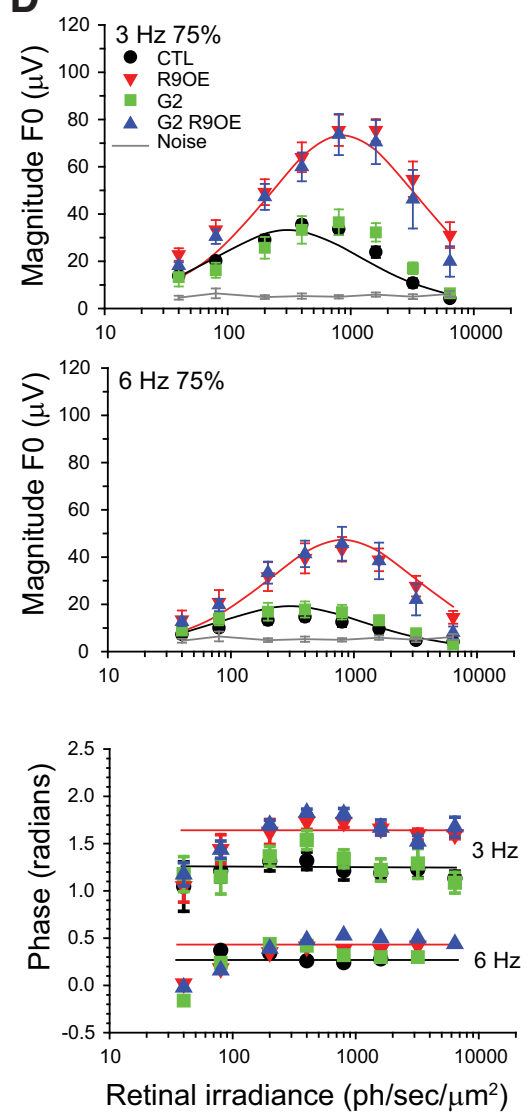

Figure 4. Higher isolated flicker ERG responses in R90E than in WT mice. A, Averages of the isolated ERG responses of WT (black line, $n=6$ ) and R90E (red line, $n=6)$ mice to $3 \mathrm{~Hz}$ (left) and 6 $\mathrm{Hz}$ (right) sinusoidal flicker stimulation of constant contrast (75\%) after intravitreal injection of glutamate receptor analogues. Flicker was presented at low $\left(40 \mathrm{ph} / \mathrm{s} / \mu \mathrm{m}^{2}\right)$, intermediate $(800$ $\mathrm{ph} / \mathrm{s} / \mu \mathrm{m}^{2}$ ), and bright $\left(6400 \mathrm{ph} / \mathrm{s} / \mu \mathrm{m}^{2}\right.$ ) retinal irradiance levels. B, Magnitude of the response to $3 \mathrm{~Hz}$ (middle plot) and $6 \mathrm{~Hz}$ (bottom plot) flicker plotted as a function of retinal irradiance for WT (black circles; $n=6$ ) and R90E (red triangles; $n=6$ ) mice. Individual responses are indicated with gray symbols. Continuous lines represent model predictions estimated with a linear-nonlinear model described in Figure 5. The steady-state suppression of the rod dark currents for WT and R90E mice (top plot) is adapted from Fortenbach et al. (2015). Note that the lateral displacement of the curves corresponds to the separation between the peaks of the magnitude plots ( 3 and $6 \mathrm{~Hz}$; vertical arrows). Statistical analysis: two-way RM ANOVA, genotype $\times$ irradiance interactions, \#p $<$ $0.05,{ }^{*} p<0.001$, all other interactions $p>0.05$. See text for details. C, Phase of the responses to 3 and $6 \mathrm{~Hz}$ flicker plotted as a function of retinal irradiance for WT (black symbols; $n=6$ ) and R90E (red triangles; $n=6$ ) mice. For clarity purposes the phase values have been displaced vertically but the relative separation between the phase values of WT and R90E was maintained. Continuous lines indicate the predicted phase values estimated from the model presented in Figure 5. Open symbols represent individual responses. Phase responses to $6 \mathrm{~Hz}$ were estimated from fits to average traces in $\boldsymbol{A}$. $\boldsymbol{D}$, Average response magnitudes for G2 (green symbols, $n=6$ ) and G2::R90E (blue symbols, $n=6$ ) mice to 3 (top) and $6 \mathrm{~Hz}$ (middle) flicker with $75 \%$ contrast plotted as a function of retinal irradiance. Data for WT and R90E mice are the same as in $B$. There is no statistical difference between WT and G2 to $3 \mathrm{~Hz}$ (two-way RM ANOVA, $p=0.6$ ) or $6 \mathrm{~Hz}$ flicker (two-way RM ANOVA, $p=0.6$ ) or between R90E and G2::R90E mice to $3 \mathrm{~Hz}$ (two-way RM ANOVA, $p=0.27$ ) or $6 \mathrm{~Hz}$ (two-way RM ANOVA, $p=0.86$ ). Red and black continuous lines represent fit of WT and R90E magnitudes with the four-parameter LNL model (same as Fig. 4B). Bottom, Phase of the responses to 3 and $6 \mathrm{~Hz}$ flicker plotted as a function of retinal irradiance. Phase of R90E and G2::R90E mice match closely. Continuous lines indicate the predicted phase values as in C. All symbols represent mean \pm SEM.

inherent to rod photoreceptor responses (Shapley and EnrothCugell, 1984). However, this notion does not explain the higher ERG response amplitudes in R9OE versus WT mice in relation to their respective rod response kinetics. We turned to a mathematical model to gain insights into the underlying mechanisms.

\section{The photoresponse recovery time-constant controls the magnitude and phase of the pharmacologically isolated ERG flicker responses}

We considered whether a simple model consisting of a single integrating stage characterized by an exponential decay function followed by a saturating nonlinearity (Fig. 5A) can explain our ERG data in Figure 4. We tentatively assign the linear integrating stage to the rod phototransduction cascade while the nonlinear function relates the suppression of the steady dark current with retinal irradiance (see Discussion). Although more sophisticated and realistic models of phototransduction (Hamer, 2000; Astakhova et al., 2015) and ERGs (Robson and Frishman, 2014) have been developed, different versions of linear-nonlinear (LNL) models have been used in the past to investigate the responses of retinal neurons to flicker and noise stimulation in electrophysiology (Tranchina et al., 1984; Rieke, 2001; Smith et al., 2001; Wang et al., 2011; Sinha et al., 2017) and psychophysics (Kelly, 1971; Watson, 1986; Stockman et al., 2006).

The model has three parameters defined as the time constant of the first order integrating reaction (Tau), the gain of the integrating stage $(h o)$ and the mean irradiance at half maximum of the saturating nonlinearity $\left(E C_{50}\right.$; Fig. 5). Solution to the equations describing the LNL model (see Materials and Methods; Eq. 14) reveals that the frequency response depends strongly on irradiance levels (Fig. 5). At low light levels (Fig. 5Ai) the family of response functions behaves as predicted by a linear model (see figure legend for details); however, as irradiance levels increase, the order of the curves reverses, such that the response curve corresponding to the shortest time constant yields the highest response amplitude (Fig. 5Aii). Furthermore, the magnitude of 


\section{A Linear-non-linear model}
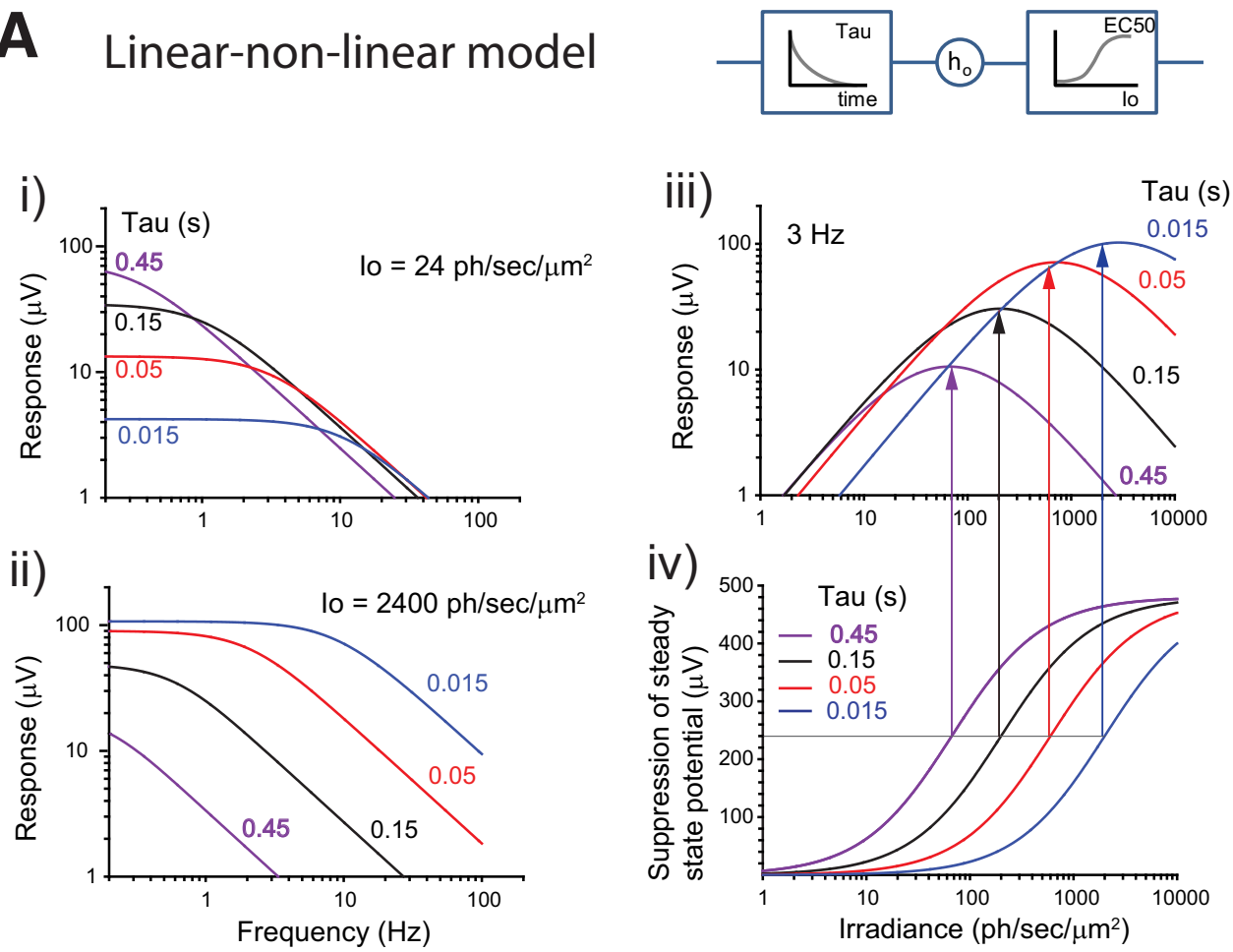

B
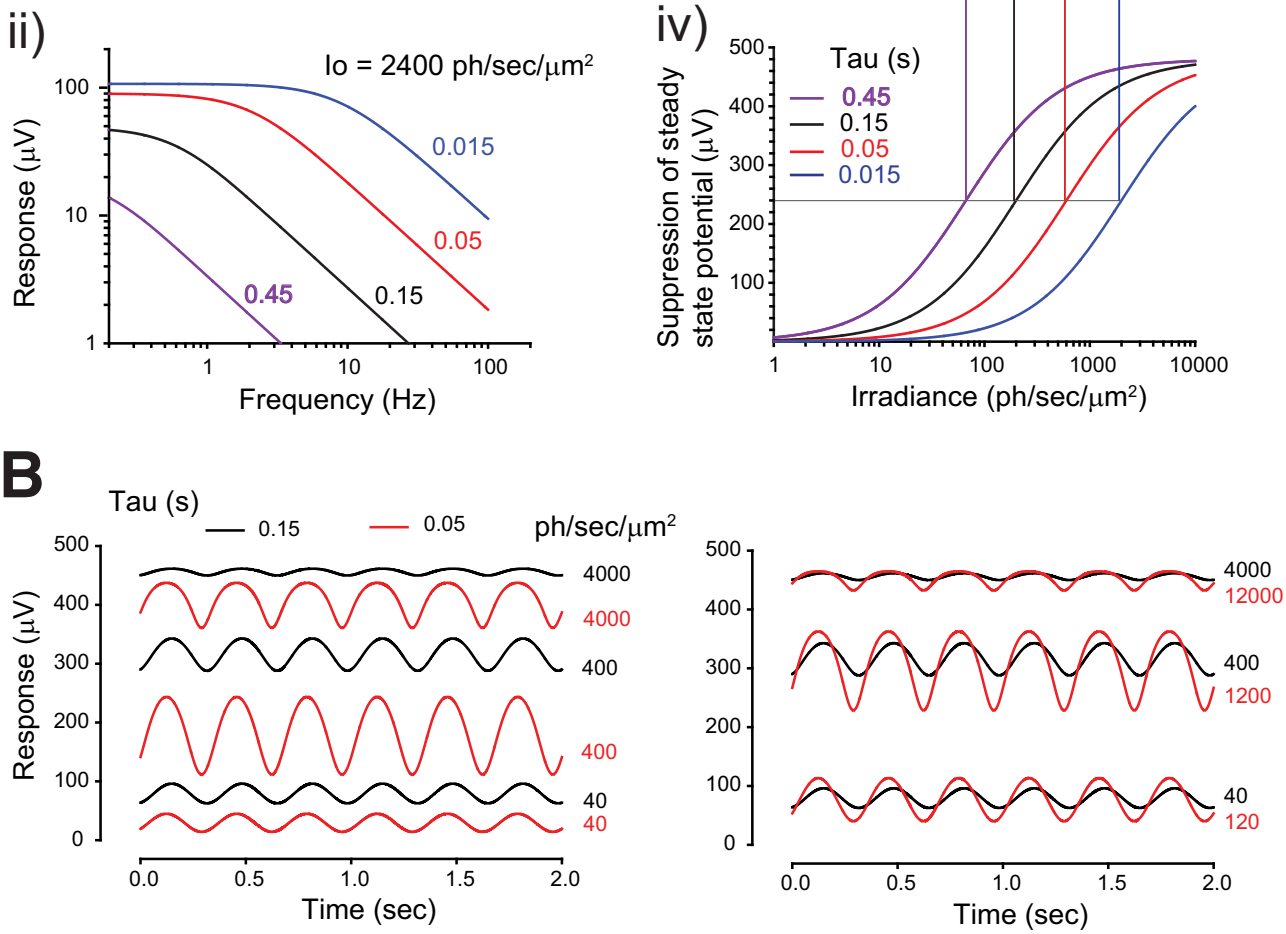

Figure 5. The photoresponse time-constant plays two distinct roles in the control of flicker responses. $\boldsymbol{A}$, Family of responses to flicker of the LNL model (see Materials and Methods; Eq. 14) plotted as a function of frequency at $(\boldsymbol{A i})$ low $\left(24 \mathrm{ph} / \mathrm{s} / \mu \mathrm{m}^{2}\right)$ and (Aii) high $\left(2400 \mathrm{ph} / \mathrm{s} / \mu \mathrm{m}^{2}\right)$ irradiance levels; values of the integrating time constants are indicated in the plots. The family of low pass functions in $A$ i resembles the tradeoff between amplitude and bandwidth of the response that is characteristic of linear systems as the integrating reaction speeds up (or equivalently, as the time constant decreases in value; Oppenheim et al., 1983). All the curves ultimately share the same high-frequency asymptote, consistent with the notion that the bandwidth increases as the time constant decreases. The amplitude of the response is proportional to the time constant, whereas the cutoff frequency (bandwidth) of the low-pass filter is inversely proportional to the time constant. Aiii, Corresponding family of responses to $3 \mathrm{~Hz}$ flicker plotted a function of irradiance and (Aiv) estimated suppression of the steady-state potential as a function of irradiance. The time constant controls the location of the compressive nonlinearity (Aiv), such that as time constant increases, the compressive nonlinearity shifts along the axis, and determines the magnitude of the response to flicker (Aiii). The vertical arrows indicate the relationship between the irradiance values eliciting $50 \%$ suppression of the steady potentials and the peak response magnitudes. See text for details. Model parameters were ho $=8 \mathrm{~s}^{-1}, \mathrm{EC}_{50}=220 \mathrm{ph} / \mathrm{s} / \mu \mathrm{m}^{2}, K=480 \mu \mathrm{V}$. B, Comparison of response time course to $3 \mathrm{~Hz}$ flicker, $75 \%$ contrast, of LNL models with slow (0.15 s; black traces) and fast $(0.05 \mathrm{~s}$; red traces) time constants as a function of irradiance (left) and after matching irradiance levels to elicit equal steady state (right).

the response to flicker follows a non-monotonic relation with mean irradiance (Fig. 5Aiii), where both the magnitude and position of the peak response are inversely related to the time constant (see Materials and Methods; Eqs. 18 and 19). Note that the position of the peak response along the abscissa corresponds to the mean irradiance level producing a $50 \%$ (steady-state) suppression of the steady potential (Fig. 5Aiv), whereas the position of the compressive nonlinearity itself is inversely related to the area under the exponential function (Eq. 14). In this scenario the response time constant plays two distinct roles (1) sets the cutoff frequency (or bandwidth) of the linear filter that shapes the sinusoidal signal, and (2) controls the location along the irradiance axis of the compressive nonlinearity that determines the magnitude of the response to flicker.

We compared the time course of the responses to flicker of our LNL model with a slow integration time $(T a u=0.15 \mathrm{~s})$ with those of a model with fast integration time ( $\mathrm{Tau}=0.05 \mathrm{~s})$. These values were chosen as an approximation to the dominant time constant values, 0.128 and $0.05 \mathrm{~s}$, that characterize the response kinetics of WT and R9OE rods, respectively (Peinado Allina et al., 2017; see Discussion). The predicted responses (Fig. $5 B$, left) capture several features we observed in the ERG responses to flicker stimulation of constant contrast (Fig. 4A): (1) at low irradiance levels ( $40 \mathrm{ph} / \mathrm{s} / \mu \mathrm{m}^{2}$, within the linear range) the two models exhibited 
responses of similar amplitude, (2) as irradiance levels rise (400 $\left.\mathrm{ph} / \mathrm{s} / \mu \mathrm{m}^{2}\right)$ the amplitude of the response of the fast model (Tau $=0.05 \mathrm{~s}$ ) was consistently higher than that of the slow model $($ Tau $=0.15 \mathrm{~s}),(3)$ at high irradiance levels $(4000 \mathrm{ph} / \mathrm{s} /$ $\mu \mathrm{m}^{2}$ ) the amplitude of the responses decreases as expected from compressive saturation, (4) a phase advance as inferred from the temporal shift in the response of the fast versus the slow models (Eq. 6). The predicted responses of the fast model remain larger than those of the slow model after adjusting the background levels (by a factor of 3 ) to elicit equal steady states (Fig. 5B, right). The position of the nonlinearity along the irradiance axis is inversely proportional to the time constant (Eqs. 12 and 14), hence, the factor of 3 follows from the threefold ratio in Tau values used for illustration of the model predictions.

Next we applied maximum likelihood estimation (Millar, 2011) to evaluate how well the LNL model (Eq.14) captures the major features of the data in Figure 4. We considered four different nested models, each with an increasing number of constraints, to identify the parameters that account for the differences in the magnitude of the response between WT and R9OE mice (Fig. 6A-D). Our analysis indicates that a model with four independent parameters is sufficient to explain the data (Fig. $6 E-G$ ) as is illustrated by the model predictions in Figure 4, $B$ (magnitude) and $C$ (phase). In this case, WT and R9OE share the same values for ho (ho-WT $=$ ho-R9OE $\left.=3.46 \pm 0.41 \mathrm{~s}^{-1}\right)$ and $\mathrm{EC}_{50}\left(\mathrm{EC}_{50}-\mathrm{WT}=\right.$ $\mathrm{EC}_{50}-\mathrm{R} 9 \mathrm{OE}=190.8 \pm 21.1 \mathrm{ph} / \mathrm{s} / \mu \mathrm{m}^{-2}$ ) but differ in the value of Tau $($ Tau-WT $=0.181 \pm 0.005 \mathrm{~s}$, Tau-R9OE $=0.070 \pm 0.003 \mathrm{~s})$. Note that these tau values are in line with the dominant time constants of WT and R9OE rods (see Discussion). We conclude that the differences in the values of the time constants (Tau) account, to a large degree, for the differences in the magnitude of the response of WT and R9OE mice.

The linear stage of the model predicts that the bandwidth of the response will increase as the time constant decreases (Fig. 5A). However, we could not empirically test this prediction because signal-to-noise limitations in our ERGs prevented us from reliably recording responses to flicker frequencies $>6 \mathrm{~Hz}$. In its place we analyzed the relative changes in response phase which is directly linked to the cutoff frequency (bandwidth) via the activation rates (or time constants) of a first-order integration function (see Eq. 6; Oppenheim et al., 1983). We found that the fourparameter model can also account reasonably well for the phase advance between the sinusoidal responses of WT and R9OE mice (Fig. 4C), particularly at irradiance levels $>100 \mathrm{ph} / \mathrm{s} / \mu \mathrm{m}^{2}$, which is in line with the prediction that the flicker ERG responses of R9OE mice have a wider bandwidth than those of WT mice.

\section{Similar isolated-ERG responses to $6 \mathrm{~Hz}$ flicker in R9OE and WT mice at behaviorally-determined contrast thresholds} We demonstrated that R9OE mice exhibit higher TCS to $6 \mathrm{~Hz}$ flicker than WT mice (Fig. 2) and also that the pharmacologicallyisolated ERG response to $75 \%$ contrast flicker is larger in $\mathrm{R} 9 \mathrm{OE}$ than in WT mice (Fig. 4). Next we investigated the correspondence between the ERG response and TCS in WT and R9OE mice (Frishman and Robson, 1999; Naarendorp et al., 2001). The goal was to determine whether the different contrasts required to reach behavioral thresholds (same sensory experience) produce the same isolated ERG response (neural encoding) in WT and R9OE mice (Parker and Newsome, 1998; Carandini and Churchland, 2013).

We measured and compared the pharmacologically-isolated ERG responses of WT and R9OE mice to flicker contrast set at the level of their respective behavioral thresholds (Fig. 7A). Contrast thresholds were computed as the inverse of the TCS values in Figure 2D (see Materials and Methods). The behavioral contrast thresholds of WT mice remained relatively constant (50-60\%) with background irradiance. Contrast thresholds of R9OE were significantly lower than those of WT mice for irradiance values $>40 \mathrm{ph} / \mathrm{s} / \mu \mathrm{m}^{2}$ (significant interaction between genotype and irradiance, $p<0.001$, power $=0.9$; two-way RM ANOVA). Note that all comparisons of WT responses within irradiance were not significantly different $(p>0.35)$, consistent with Weber adaptation in WT mice.

The ERG responses of WT and R9OE mice to flicker set at the corresponding contrast thresholds matched closely over the entire irradiance range (Fig. $7 B$ ). The magnitude of the fundamental flicker ERG responses in WT and R9OE mice increased approximately linearly with irradiance up to $800 \mathrm{ph} / \mathrm{s} / \mu \mathrm{m}^{2}$ (Fig. $7 C$ ), and decreased monotonically thereafter, most likely the result of response compression (Figs. 4, 5). The close overlap of the flicker response magnitudes [no significant interaction between genotype and frequency ( $p=0.075)$ or difference among the two genotypes ( $p=0.34)$; two-way RM ANOVA] indicates that the isolated ERG responses (neural encoding) in WT and R9OE mice are similar at their respective behavioral contrast thresholds (same sensory experience).

\section{R9AP overexpression in rods increases whole ERG responses to both low and high temporal frequencies}

The amplitude of the pharmacologically-isolated ERGs declines sharply with temporal frequency, limiting the recording range to $6 \mathrm{~Hz}$. To investigate the retinal responses to higher frequencies we recorded the flicker ERG responses in eyes that were not injected with pharmacological blockers (full ERG). This signal reflects the activity of second-order bipolar cells (Shirato et al., 2008). Using this approach, we reliably recorded ERG responses to flicker frequencies ranging from 3 to $24 \mathrm{~Hz}$ at increasing irradiance levels (Fig. 8A). WT and R9OE mice had similar ERG magnitudes at mean retinal irradiances ranging from 10 to $\sim 100-200 \mathrm{ph} / \mathrm{s} /$ $\mu \mathrm{m}^{2}$. At irradiance levels $>200 \mathrm{ph} / \mathrm{s} / \mu \mathrm{m}^{2}$ the responses of R9OE mice were significantly higher than those of WT mice across the entire frequency range tested, including the response to $24 \mathrm{~Hz}$ flicker (for details of the statistical analysis, see Fig. 8).

Plots of response magnitude as a function of frequency were low-pass in shape, without the characteristic low-frequency rolloff that is observed in the full ERGs when mice are exposed to significantly higher, photopic light levels (Krishna et al., 2002; Shirato et al., 2008). The bandwidth and magnitude of the plots depended on genotype, flicker contrast, and mean irradiance level (Fig. 8B) as reported by Fortenbach et al. (2015). At 80 $\mathrm{ph} / \mathrm{s} / \mu \mathrm{m}^{2}$ the magnitude plots of WT and R9OE mice overlapped closely, whereas at a higher irradiance delivering $1600 \mathrm{ph} / \mathrm{s} / \mu^{2}$ at the retina, the responses of R9OE were $\sim 2.2$-fold higher than those of WT mice. The larger magnitude of the full ERG responses probably reflects the larger responses of ON and OFF bipolar cells in R9OE relative to WT mice (Fortenbach et al., 2015). Similar results were observed both with 75 and 25\% contrast flickers. We conclude that R9AP-overexpression in rods enhances the magnitude of the full ERG responses across the temporal frequency range (up to $36 \mathrm{~Hz}$ ) for retinal irradiance levels $>100-200 \mathrm{ph} / \mathrm{s} / \mu \mathrm{m}^{2}$.

The similarity of the full ERG response magnitudes at the lower irradiance levels $<100-200 \mathrm{ph} / \mathrm{s} / \mu \mathrm{m}^{2}$ for R9OE and WT mice (Fig. 8) follows the similarity for their isolated ERG magnitudes (Fig. 4B, bottom) and their behavioral sensitivities (Fig. $2 D)$. However, with retinal irradiance levels $>100-200 \mathrm{ph} / \mathrm{s} /$ 
A 6 parameters

WT

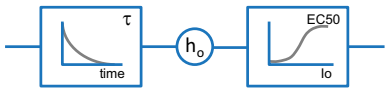

R9OE $\triangle_{\text {time }}^{\tau}$ (b)

B 5 parameters

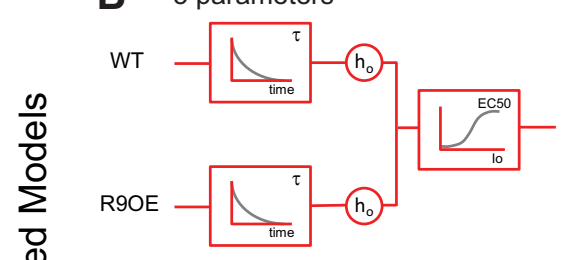

C 4 parameters

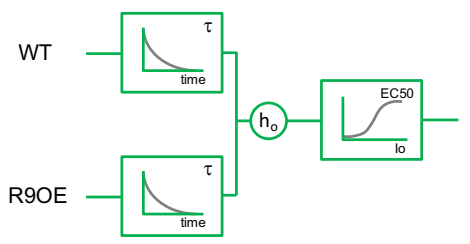

D 3 parameters

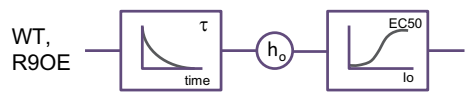

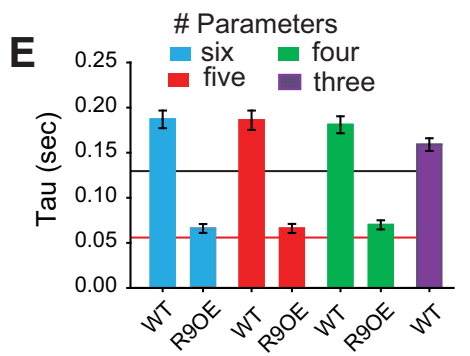

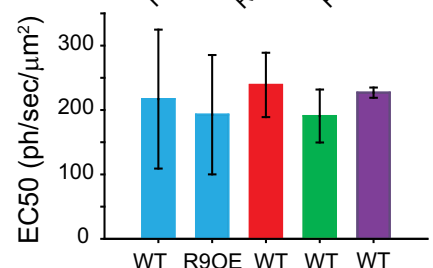

WT R9OE WT WT WT

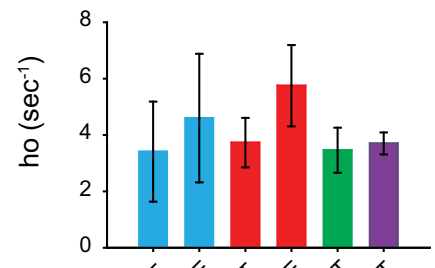

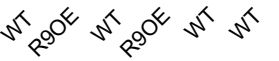

H

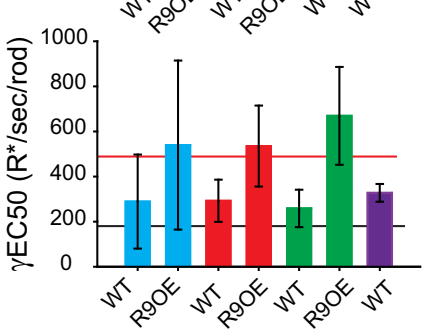

$\mathbf{F}$
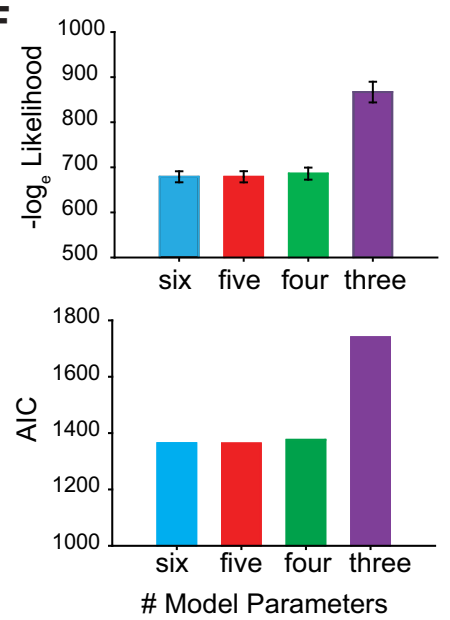

G

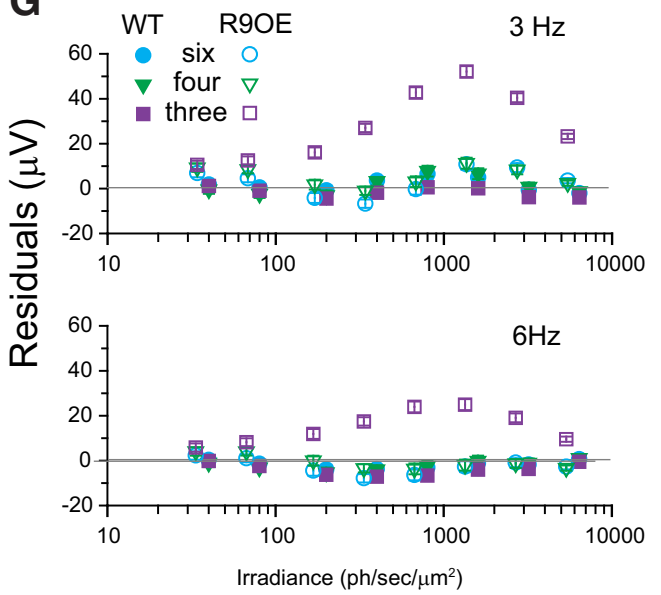

Figure 6. Differences in the values of the time constants (Tau) accounts for the differences in the magnitude of the isolated ERG response of WT versus R90E mice. $A$, Nested model with six parameters under the assumption that the values of $T a u$, ho, and $E C_{50}$ are different in WT and R90E ERG responses (parameters: Tau-WT, Tau-R90E, ho-WT, ho-R90E, EC ${ }_{50}-\mathrm{WT}$, and EC $\left.C_{50}-\mathrm{R}^{2} \mathrm{E} \mathrm{E}\right) . \boldsymbol{B}_{\text {, }}$ Nested model with five parameters tests the likelihood that $E C_{50}$ is the same in WT and R90E mice (parameters: Tau-WT, Tau-R90E, ho-WT, ho-R90E, and EC $C_{50}$-WT). $C_{\text {, Nested model with four }}$ parameters tests the likelihood that both $E C_{50}$ and ho are the same in WT and R90E mice (parameters: Tau-WT, Tau-R90E, ho-WT, and EC $C_{50}$-WT). D, Nested model with three parameters has the additional constraint that the time constants are the same in WT and R90E mice (parameters: Tau-WT, ho-WT, and EC ${ }_{50}-\mathrm{WT}$ ). E, Maximum likelihood estimation of Tau, ho, and $E C_{50}$ using the different nested models. Values were optimized by simultaneously fitting the data in Figure $4 B$ for R9OE $(n=6)$ and WT $(n=6)$ responses to two frequencies ( 3 and $6 \mathrm{~Hz}$ ) over the entire irradiance range tested (8 irradiance levels). Parametric bootstrapping was applied to determine the variability in the parameter estimates. Error bars represent $95 \%$ confidence intervals. The values of the parameters in the nested models did not change significantly from those in the unrestricted model (6 parameters), as can be readily inferred from the overlap of their respective confidence intervals. All calculations were performed with MATLAB (MathWorks). Black and red lines represent the dominant time constant values for WT and R90E rods, respectively, as determined by Chen et al. (2010) and by Peinado Allina et al. (2017). F, Log Likehood (top) and Akaike information coefficient (AIC; bottom) for the nested models. The log likelihood indicates the probability that the data were generated by the nested models, while the AIC was used to compare the different models by taking into consideration the number of free parameters. As can be inferred from the plot, nested models with six, five, and four parameters had similar AIC values, suggesting equally good fits of the data. However, the nested model with three parameters resulted in a much higher AIC value indicating a poor fit to the data relative to that provided by the other models and consistent with the notion that the data could not arise from WT and R90E mice with similar rod response kinetics. $G$, Residuals following fit to $3 \mathrm{~Hz}$ (top) and $6 \mathrm{~Hz}$ (bottom) data in Figure 4 with nested models. Visual inspection of the residuals for the fitted models reveals that models with six $\left(R^{2}=0.57, \mathrm{RMSE}=26.7 \mu \mathrm{V}\right)$ and four $\left(R^{2}=0.57, \mathrm{RMSE}=25.6 \mu \mathrm{V}\right)$ parameters provide a closer fit to the data than the model with three parameters $\left(R^{2}=-0.75, \mathrm{RMSE}=51.4 \mu \mathrm{V}\right)$. The residuals also indicate that nested models with six, five (data not shown), and four parameters produced similar fits with a small but systematic overestimation of the responses to $3 \mathrm{~Hz}$ flicker and an underestimation of the responses to $6 \mathrm{~Hz}$. Such systematic departure of the residuals from baseline suggests that the models fall short of accounting for all the possible variables (e.g., the effects of light adaptation on response kinetics have not been considered). Despite these shortcomings, we can conclude that differences in the values of the time constants (tau) in the nested model with four parameters sufficiently accounts for the differences in the magnitude of the response of WT versus R90E mice. $\boldsymbol{H}$, Values of $\gamma \mathrm{EC}_{50}$ for WT and R90E mice estimated with the different nested models (Eq. 14). The scaling factor $\gamma$ represents the lateral shift (along the irradiance axis) of the static nonlinearity and is proportional to the inverse of the exponential integration area (Eq. 12). Also shown are the $E C_{50}$ values for the nonlinear suppression of the steady dark current measured from isolated WT (184 R*/rod/s; black line) and R9AP overexpressing rods (480 R*/rod/s; red line; Fortenbach et al., 2015). Error bars are the respective $95 \%$ confidence intervals. Note that to express the $\gamma \mathrm{EC}_{50}$ values in terms of photo-isomerizations we scaled the retinal irradiance values by $0.85-0.87 \mu \mathrm{m}^{2}$, the end-on rod collecting areas (Lyubarsky et al., 2004; Naarendorp et al., 2010).

$\mu \mathrm{m}^{2}$, a more complex, frequency-dependent scenario emerges between R9OE mice responses relative to those of WT mice. In response to low frequencies (e.g., $6 \mathrm{~Hz}$ ), we observe a significant increase in the full ERG (Fig. 8A), as in the isolated ERG (Fig. 4, bottom), and the behavioral contrast sensitivity (Fig. 2D). In response to high frequencies (e.g., $21-24 \mathrm{~Hz}$ ), the magnitude of the full ERG response in R9OE mice is also larger than that of WT mice (Fig. 8A) but this enhanced response does not translate into a corresponding increase in their temporal contrast sensitivities (Fig. 2E). In summary, the results indicate that both low and high-frequency flicker responses are enhanced in R9OE retinas, but only low-frequency flicker results in a higher temporal contrast sensitivity. Furthermore, these results suggest that the frequency-dependent differences in the 

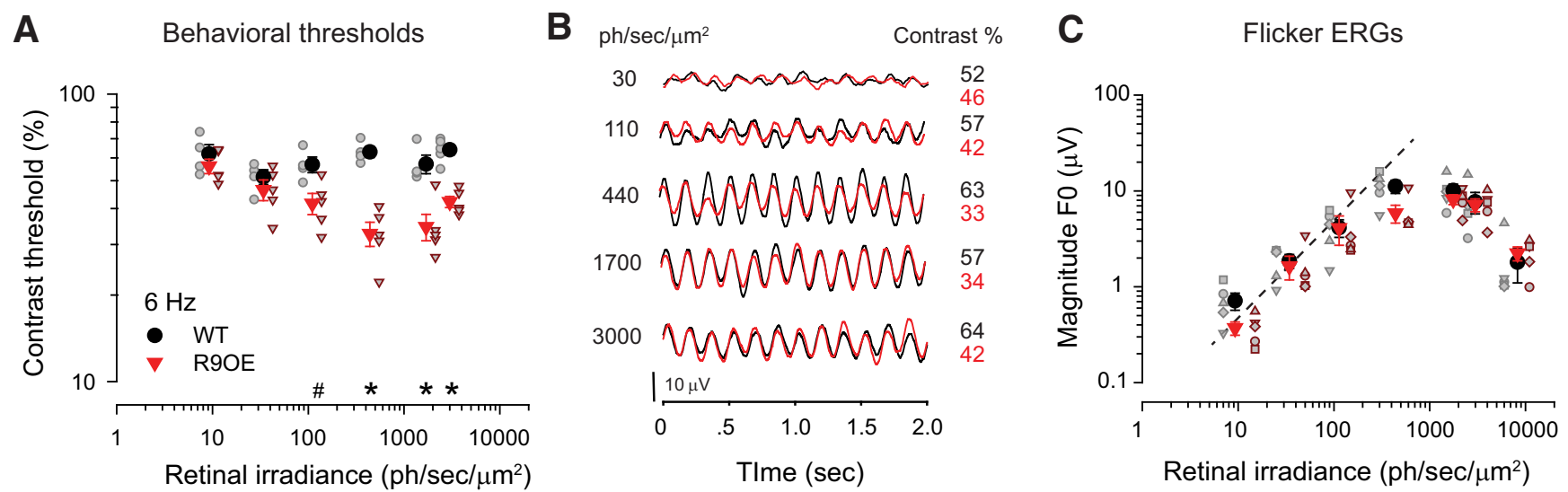

Figure 7. Similar isolated-ERG responses to $6 \mathrm{~Hz}$ flicker in R90E and WT mice at behaviorally-determined contrast thresholds. $A$, Temporal contrast thresholds of WT (black circles; averages \pm $\mathrm{SEM}, n=4$ ) and R90E (red triangles; $n=5$ ) plotted as a function of retinal irradiance in response to $6 \mathrm{~Hz}$ flicker stimulation. Contrast thresholds were calculated from the TCS data in Figure $2 D$ by applying the relation threshold $=100 \times(\mathrm{TCS})^{-1}$. The gray symbols are the individual responses and have been displaced laterally for illustration purposes. $\boldsymbol{B}$, Averages of the pharmacologicallyisolated ERG responses of WT (black traces; $n=5$ ) and R90E (red traces; $n=5$ ) mice to $6 \mathrm{~Hz}$ sinusoidal flicker stimulation with mean retinal irradiance levels indicated to the left and contrast values indicated to the right of the traces (according to color code). The contrast values used for the flicker ERG stimulus are the same as the contrast thresholds determined behaviorally at the corresponding mean irradiance level. C, Fundamental magnitude of the isolated flicker ERG responses of WT (black circles; $n=5$ ) and R90E (red triangles; $n=5$ ) mice to flicker set at the behavioral contrast threshold values indicated in $A$ and plotted as a function of the corresponding mean retinal irradiance. No statistical differences were detected: two-way RM ANOVA. See text for details. Dashed line with unitary slope, fit by eye, illustrates the approximately linear increase of the ERG responses at irradiance levels $<400 \mathrm{ph} / \mathrm{s} / \mu \mathrm{m}^{2}$. Error bars indicate SEM. Statistical analysis: two-way RM ANOVA, irradiance $x$ genotype interactions, $\# p<0.05,{ }^{*} p<0.001$, all other interactions $p>0.05$.
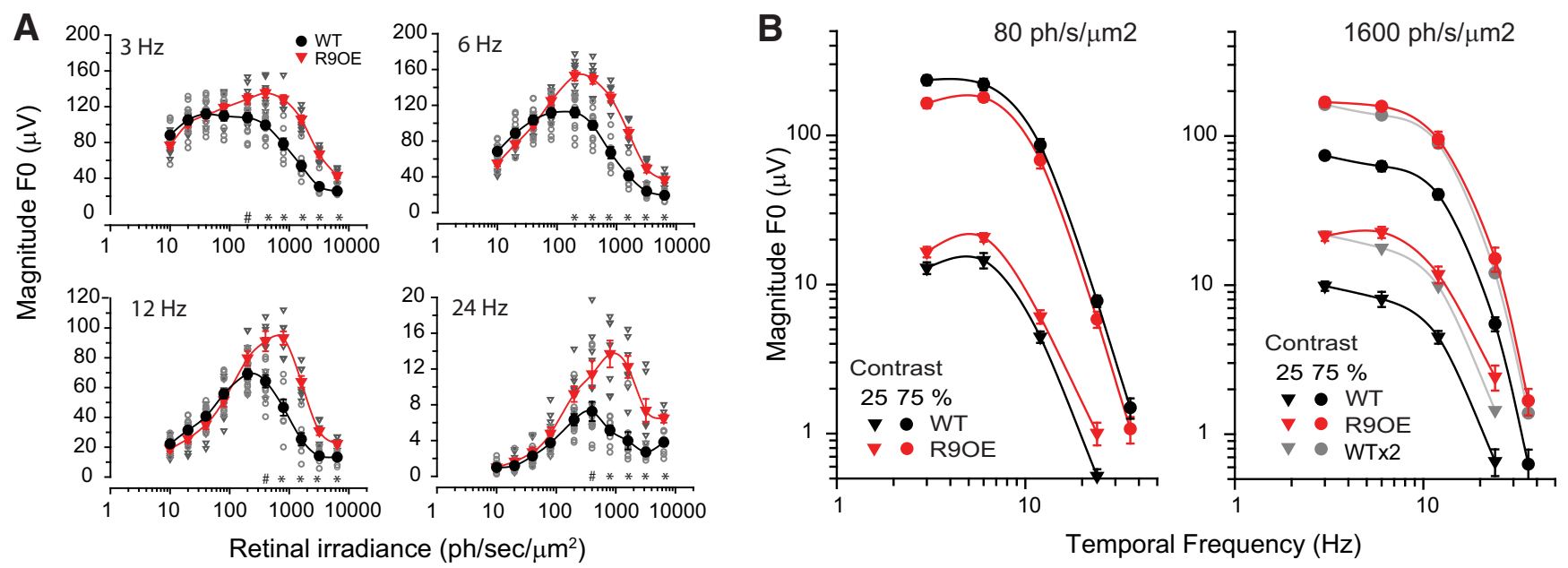

Figure 8. Whole ERG responses are larger in R9OE than in WT mice at retinal irradiance levels $>200 \mathrm{ph} / \mathrm{s} / \mu \mathrm{m}^{2}$. A, Fundamental magnitude of the full (without injection of blockers) ERG responses of WT (black circles; $n=6$ ) and R90E (red triangles; $n=5$ ) mice to $75 \%$ flicker plotted as function irradiance. Top to bottom, plots show the magnitudes in response to flicker frequencies $3,6,12$, and $24 \mathrm{~Hz}$ respectively. Statistical analysis: two-way RM ANOVAs performed independently at each flicker frequency yield significant genotype $\times$ retinal irradiance interactions $(p<0.001)$. Pair wise comparison $p$ values (Holm-Sidak method) are indicated in the plots, where \#p $<0.02$ and ${ }^{*} p<0.001$; for all others $p>0.05$. Open gray symbols are individual responses; filled symbols are mean values \pm SEM. $\boldsymbol{B}$, Magnitude spectrum for WT (black symbols; $n=4-5$ ) and R90E (red symbols; $n=4-5$ ) measured at two mean retinal irradiance levels: 80 (left) and 1600 (right) $\mathrm{ph} / \mathrm{s} / \mu \mathrm{m}^{2}$. Flicker contrast was $25 \%$ (circles) and $75 \%$ (triangles). Filled gray symbols represent the magnitudes of the WT responses at $1600 \mathrm{ph} / \mathrm{s} / \mu \mathrm{m}^{2} \mathrm{scaled} \mathrm{by} 2.2$.

processing of temporal flicker may arise downstream of the bipolar cells, where the full ERG signals arise (see Discussion for candidate mechanisms).

\section{Discussion}

In this study we applied an operant behavior assay, electrophysiological recordings, and mathematical modeling to investigate the retinal underpinnings of TCS in mesopic lights. We have determined that photoresponse rate recovery in rods is critical in setting TCS to low but not high, temporal frequencies. These results have important implications in our understanding of the retinal mechanisms that limit temporal sensitivity at different light levels and provide an unexpected, novel mechanistic perspective on Weber's law.

\section{Rod photoresponse recovery kinetics limit TCS in the Weber regime}

We have previously shown that R9AP KO transgenic mice with slow rod and cone photoresponse recovery kinetics exhibit a reduction in the bandwidth of their optomotor response at mesopic light levels ( $1600 \mathrm{R}^{\star} / \mathrm{rod} / \mathrm{s}$ ) relative to control WT mice (Umino et al., 2012). Our previous results are similar to those observed in the human condition, bradyopsia, in which humans respond slowly to changes in light levels. However, those results do not demonstrate that rod kinetics are the rate-limiting stage in mesopic lights. Specifically, a non-limiting stage in a serial system can be slowed down to the point where it becomes the ratelimiting element of the system. In contrast, a stage is considered 
as being rate limiting if speeding up its kinetics results in faster responses (and implicitly an extended bandwidth) of the system, as in the context of the phototransduction pathway (Krispel et al., 2006). In our previous study (Umino et al., 2012), we did not examine optomotor response sensitivities of mice with fast photoresponse kinetics at mesopic light levels.

Here, our first major finding is that overexpression of the transducin GAP complex in rods of R9OE mice increased behavioral TCS to low $(6 \mathrm{~Hz})$ but not high $(21 \mathrm{~Hz})$ temporal frequencies at retinal irradiance levels ranging from 100 to $4000 \mathrm{ph} / \mathrm{s} /$ $\mu \mathrm{m}^{2}$. These light levels correspond to the mesopic range in mouse vision (Nathan et al., 2006; Naarendorp et al., 2010). This is also the range where mouse TCS exhibits rod-driven Weber adaptation in response to flickering lights (Figs. 2, 3). In the context of TCS functions in human observers, Weber adaptation is apparent in the low-frequency asymptote, where TCS remains approximately constant as retinal irradiance values rise (de Lange Dzn, 1958; Kelly, 1961; Conner, 1982). Weber behavior depends on the spatial configuration of the stimulating pattern (Kelly, 1972) and is thought to reflect the inhibitory influences of feedback mechanisms acting in the inner and outer retinas (Kelly, 1971; Shapley and Enroth-Cugell, 1984; Tranchina et al., 1984; Purpura et al., 1990; Seiple et al., 1992; Dunn et al., 2007).

At a physiological level, Weber adaptation is observed in photoreceptor (Matthews et al., 1988), synaptic (Oesch and Diamond, 2011), and postsynaptic responses (Barlow, 1965; Dunn et al., 2006). However, it remains unclear as to how the different retinal components and circuits combine to produce Weber-like behavior. Here we have established that rod photoresponse kinetics limit TCS in the Weber regime: faster rod photoresponse kinetics in R9OE mice resulted in an increase in TCS (Figs. 2,3) and flicker ERG responses (Fig. 4) relative to WT mice. Our results may appear counterintuitive at first glance, because faster response kinetics in rate-limiting reactions is associated with a reduction of the integration times and smaller responses (Fig. 5). However, the higher contrast sensitivity in the responses of R9OE versus WT mice can be explained in terms of a shift in a compressive nonlinearity that we tentatively assign to the steady-state activation of the cGMP-gated channels in rods (see below).

We established that behavioral thresholds elicit similar pharmacologically-isolated ERG responses in WT and R9OE mice to $6 \mathrm{~Hz}$ flicker (Fig. 7) suggesting a close correspondence between the neural retinal signal and the behavioral response. However, the isolated ERG response reflects both the contributions of the photoreceptor response as well as contributions of the slow PIII (and perhaps a c-waveform) originating from the flow of potassium currents in Muller (and RPE) cells (Shirato et al., 2008; Robson and Frishman, 2014). Surprisingly, the estimated parameters in the model are in close agreement with physiologically measured values in rod photocurrents. The model time constants for WT and R9OE ERGs were slightly slower but in line with the values of the dominant time constants of WT $(0.128 \mathrm{~s}$; Fig. $6 E$, black line) and the two different lines of mice overexpressing R9AP in rods (Chen et al., 2010; Peinado Allina et al., 2017; 0.058 s; Fig. $6 E$, red line). Similarly, the values of the product $\gamma \mathrm{EC}_{50}$, representing the shift of the static nonlinearity along the irradiance axis for WT and R9OE rods (Fig. 6H; Eq. 14), are well within range of the $E C_{50}$ values of the steady circulating currents measured in isolated WT $(184 \mathrm{R} * / \mathrm{rod} / \mathrm{s})$ and R9AP overexpressing rods (480 R/rod/s; Fortenbach et al., 2015). Hence, we tentatively assign the linear integrating stage of the model to the rod phototransduction cascade while the nonlinear function relates the suppression of the steady dark current with retinal irradiance. Future studies are needed to precisely determine the relative contribution and kinetics of photoreceptors, Muller and RPE cells to the isolated ERG, with particular interest in the individual contributions at different light levels and temporal frequencies.

An important outcome of our studies is the similarity in the behavioral TCS in R9OE and WT mice at weak irradiance levels that seems to follow what occurs in the isolated and full ERG magnitudes. We applied our mathematical model to gain new insights into why photoreceptor response kinetics do not seem to influence TCS at dim, scotopic light levels $\left(40-100 \mathrm{ph} / \mathrm{s} / \mu \mathrm{m}^{2}\right.$, when rods integrate the response of successive photo-isomerizations) as observed here (Figs. 2-4, 8) as well as by Peinado Allina et al. (2017) using a different operant (running wheel) assay. Inspection of Figure $5 A i$ shows that the simulated responses to 3 and $6 \mathrm{~Hz}$ flicker of WT ( Tau $=0.150 \mathrm{~s}$ ) and R9OE $(\mathrm{Tau}=0.05 \mathrm{~s})$ photoreceptors share the same asymptote, resulting in similar response magnitudes at retinal irradiance levels of $24 \mathrm{ph} / \mathrm{s} / \mu^{2}$ (at these irradiance levels the model predicts a relatively linear response in the temporal frequency domain). These predictions seem to bear out in the isolated ERG responses to 6 $\mathrm{Hz}$ flicker and approximately in the case of $3 \mathrm{~Hz}$ (Fig. 4), suggesting that the behavioral and full ERG phenotype can be attributed to the photoreceptor responses. Our argument is based on the premise that rods integrate the responses of multiple photoisomerizations at these light levels, as can be inferred from the recordings by Fortenbach et al. (2015), which show rod dark current suppression levels of $\sim 20 \%$ in WT and $\sim 10 \%$ in R9OE rods at $40 \mathrm{R}^{\star} / \mathrm{rod} / \mathrm{s}$. We note however, that these results do not rule out the possibility that elements downstream from the retinal bipolar cells may limit the rate of the responses as proposed by Peinado Allina et al. (2017).

\section{Rod photoresponse recovery kinetics do not limit temporal resolution}

Our second major finding in this study is that TCS to high frequencies $(21 \mathrm{~Hz})$ was not affected when speeding up rod photoresponse kinetics (Figs. 2, 3). Interestingly Weber adaptation does not apply to the sharp, high-frequency asymptote of the TCSFs (Kelly, 1972; Shapley and Enroth-Cugell, 1984; Watson, 1986). To this day it remains unknown why and how the visual system processes flicker information differentially in the low (Weber) and high (linear) frequency regions of the TCSFs. It may be argued that the faster kinetics of R9OE rods are low-pass filtered at the rod-bipolar cell synapses and not transmitted to the inner retina. However, results from our full ERGs recordings oppose this view. The magnitude of the full ERGs responses to flicker presented at irradiance levels $>100-200 \mathrm{ph} / \mathrm{s} / \mu \mathrm{m}^{2}$ was significantly larger in R9OE than in WT mice for temporal frequencies ranging from 1.5 to $\sim 36 \mathrm{~Hz}$ (Fig. 8). Given that the magnitude of the full ERGs is related to the response of bipolar cells (Shirato et al., 2008), our results are consistent with the notion that the fast, larger signals in R9OE rod photoreceptors are being transmitted postsynaptically to the inner retina. Our results are also consistent with earlier observations showing that the full ERGs and rod bipolar cell flicker responses are larger in R9OE than in WT retinas but disagree with the irradiance range $\left(<100 \mathrm{R}^{*} / \mathrm{rod} / \mathrm{s}\right)$, where Fortenbach et al. (2015) report the increase in the responses of R9OE versus WT mice. This difference may arise as a consequence of the different light adaptation conditions applied in the two studies. The measurements performed by Fortenbach et al. (2015) were performed following short (in 
the order of seconds) steady light exposures, whereas our studies were performed following prolonged exposures $(>40 \mathrm{~min}$ in $\mathrm{du}-$ ration, aimed at replicating our behavioral experimental conditions) that may alter rod photoresponse properties through a number of light-dependent mechanisms such as pigment bleaching (Lyubarsky et al., 2004), prolonged adaptation effects (Calvert et al., 2002; Krispel et al., 2003), and/or translocation of key phototransduction components at the strongest intensities of our study (Sokolov et al., 2002; Lobanova et al., 2007).

Our next observation is that the larger response to low and high temporal frequencies at the level of the inner retina (as reflected by the full ERG) is differentially transmitted downstream in R9OE versus WT mice. As described above, the larger magnitudes in the isolated ERG and full ERG responses to low flicker frequencies $(6 \mathrm{~Hz})$ correspond to an increase in contrast sensitivity at the behavioral level of R9OE versus WT mice. However, the larger response magnitudes to high temporal frequencies (e.g., 21 $\mathrm{Hz}$ ) did not correspond to increased behavioral contrast sensitivity and/or temporal resolution. This suggests that: (1) neural mechanism(s) located downstream from the bipolar cells differentially control TCS to high (e.g., $24 \mathrm{~Hz}$ ) flicker frequencies (Abbott et al., 1997; Carandini et al., 2002; Chung et al., 2002), or (2) the relatively small differences in the retinal response of $\mathrm{R} 9 \mathrm{OE}$ versus WT mice to $24 \mathrm{~Hz}$ flicker (Fig. 8A) are below intrinsic (central) visual noise levels and cannot be detected perceptually (Pelli and Farell, 1999; Rovamo et al., 1999). In either case, it will be important to identify and characterize the retinal pathways that relay these fast signals. In scotopic conditions, rod signals are transmitted largely to rod bipolar cells along the primary pathway (Dunn et al., 2006). The primary pathway in mouse remains active as light levels rise into the mesopic range $\left(250 \mathrm{R}^{\star} / \mathrm{rod} / \mathrm{s}\right)$, however, with a limited temporal frequency range (Ke et al., 2014). Secondary (via rod-cone coupling) and tertiary pathways (via direct rod to cone bipolar cell contacts) are posited to relay fast signals in mesopic lights (for review, see Sharpe and Stockman, 1999; Grimes et al., 2018a; Rivlin-Etzion et al., 2018) although a recent study shows that the primary rod (or dominant) pathway in primates can perform this duty across the mesopic range (Grimes et al., 2018b).

In conclusion, our results establish clear differences between the scotopic and mesopic vision in mouse. Much like in primate vision, a pattern is beginning to emerge where the control of TCS shifts from the inner retina in dim lights (Robson and Frishman, 1995; Sampath et al., 2005; Stockman et al., 2010; Umino et al., 2012) to the rod kinetics in mesopic lights (Grimes et al., 2018b; results of this study). Future studies will tell whether photopic TCS in mouse relates to the temporal response properties of cones as is observed in primates (Sinha et al., 2017).

\section{References}

Abbott LF, Varela JA, Sen K, Nelson SB (1997) Synaptic depression and cortical gain control. Science 275:220-224.

Astakhova L, Firsov M, Govardovskii V (2015) Activation and quenching of the phototransduction cascade in retinal cones as inferred from electrophysiology and mathematical modeling. Mol Vis 21:244-263.

Azevedo AW, Rieke F (2011) Experimental protocols alter phototransduction: the implications for retinal processing at visual threshold. J Neurosci 31:3670-3682.

Barlow HB (1965) Optic nerve impulses and Weber's law. Cold Spring Harb Symp Quant Biol 30:539-546.

Brown TM, Allen AE, Wynne J, Paul DL, Piggins HD, Lucas RJ (2011) Visual responses in the lateral geniculate evoked by $\mathrm{Cx} 36$-independent rod pathways. Vision Res 51:280-287.

Bushnell M, Umino Y, Solessio E (2016) A system to measure the pupil response to steady lights in freely behaving mice. J Neurosci Methods 273:74-85.

Calvert PD, Govardovskii VI, Arshavsky VY, Makino CL (2002) Two temporal phases of light adaptation in retinal rods. J Gen Physiol 119:129-145.

Cao D, Lee BB, Sun H (2010) Combination of rod and cone inputs in parasol ganglion cells of the magnocellular pathway. J Vis 10(11):4 1-15.

Carandini M, Churchland AK (2013) Probing perceptual decisions in rodents. Nat Neurosci 16:824-831.

Carandini M, Heeger DJ, Senn W (2002) A synaptic explanation of suppression in visual cortex. J Neurosci 22:10053-10065.

Chang B, Dacey MS, Hawes NL, Hitchcock PF, Milam AH, Atmaca-Sonmez P, Nusinowitz S, Heckenlively JR (2006) Cone photoreceptor function loss-3, a novel mouse model of achromatopsia due to a mutation in Gnat2. Invest Ophthalmol Vis Sci 47:5017-5021.

Chen CK, Woodruff ML, Chen FS, Chen D, Fain GL (2010) Background light produces a recoverin-dependent modulation of activated-rhodopsin lifetime in mouse rods. J Neurosci 30:1213-1220.

Chen CK, Woodruff ML, Chen FS, Chen Y, Cilluffo MC, Tranchina D, Fain GL (2012) Modulation of mouse rod response decay by rhodopsin kinase and recoverin. J Neurosci 32:15998-16006.

Chung S, Li X, Nelson SB (2002) Short-term depression at thalamocortical synapses contributes to rapid adaptation of cortical sensory responses in vivo. Neuron 34:437-446.

Conner JD (1982) The temporal properties of rod vision. J Physiol 332:139_ 155.

de Lange Dzn H (1954) Relationship between critical flicker frequency and a set of low frequency characteristics of the eye. J Opt Soc Am 44:380-389.

de Lange Dzn H (1958) Research into the dynamic nature of the human fovea-cortex systems with intermittent and modulated light: I. Attenuation characteristics with white and colored light. J Opt Soc Am 48:777784.

Donner K, Copenhagen DR, Reuter T (1990) Weber and noise adaptation in the retina of the toad bufo marinus. J Gen Physiol 95:733-753.

Dowling JE (1967) The site of visual adaptation. Science 155:273-279.

Dunn FA, Doan T, Sampath AP, Rieke F (2006) Controlling the gain of rod-mediated signals in the mammalian retina. J Neurosci 26:3959-3970.

Dunn FA, Lankheet MJ, Rieke F (2007) Light adaptation in cone vision involves switching between receptor and post-receptor sites. Nature 449: 603-606.

Field GD, Rieke F (2002) Nonlinear signal transfer from mouse rods to bipolar cells and implications for visual sensitivity. Neuron 34:773-785.

Fortenbach CR, Kessler C, Peinado Allina G, Burns ME (2015) Speeding rod recovery improves temporal resolution in the retina. Vision Res 110:5767.

Frishman L, Robson JG (1999) Inner signal processing: adaptation to environmental light. In: Adaptive mechanisms in the ecology of vision (Archer SN, Djamgoz BA, Loew ER, eds). Dordrecht, The Netherlands: Klwer Academic.

Graham N, Hood DC (1992) Modeling the dynamics of light adaptation: the merging of two traditions. Vision Res 32:1373-1393.

Green DM, Swets JA (1988) Signal detection theory and psychophysics. Los Altos, California: Peninsula.

Grimes WN, Songco-Aguas A, Rieke F (2018a) Parallel processing of rod and cone signals: retinal function and human perception. Annu Rev Vis Sci 4:123-141.

Grimes WN, Baudin J, Azevedo AW, Rieke F (2018b) Range, routing and kinetics of rod signaling in primate retina. eLife 7:e38281.

Hamer RD (2000) Computational analysis of vertebrate phototransduction: combined quantitative and qualitative modeling of dark- and lightadapted responses in amphibian rods. Vis Neurosci 17:679-699.

He W, Cowan CW, Wensel TG (1998) RGS9, a GTPase accelerator for phototransduction. Neuron 20:95-102.

Hess RF, Nordby K (1986) Spatial and temporal limits of vision in the achromat. J Physiol 371:365-385.

Jackman SL, Choi SY, Thoreson WB, Rabl K, Bartoletti TM, Kramer RH (2009) Role of the synaptic ribbon in transmitting the cone light response. Nat Neurosci 12:303-310.

Jarsky T, Cembrowski M, Logan SM, Kath WL, Riecke H, Demb JB, Singer JH (2011) A synaptic mechanism for retinal adaptation to luminance and contrast. J Neurosci 31:11003-11015. 
Jarvis JR, Prescott NB, Wathes CM (2003) A mechanistic inter-species comparison of flicker sensitivity. Vision Res 43:1723-1734.

Ke JB, Wang YV, Borghuis BG, Cembrowski MS, Riecke H, Kath WL, Demb JB, Singer JH (2014) Adaptation to background light enables contrast coding at rod bipolar cell synapses. Neuron 81:388-401.

Keenan WT, Rupp AC, Ross RA, Somasundaram P, Hiriyanna S, Wu Z, Badea TC, Robinson PR, Lowell BB, Hattar SS (2016) A visual circuit uses complementary mechanisms to support transient and sustained pupil constriction. eLife 5:e15392.

Kelly DH (1961) Visual response to time-dependent stimuli: I. Amplitude sensitivity measurements. J Opt Soc Am 51:422-429.

Kelly DH (1971) Theory of flicker and transient responses: I. Uniform fields. J Opt Soc Am 61:537-546.

Kelly DH (1972) Adaptation effects on spatio-temporal sine-wave thresholds. Vision Res 12:89-101.

Kondo M, Sieving PA (2001) Primate photopic sine-wave flicker ERG: vector modeling analysis of component origins using glutamate analogs. Invest Ophthalmol Vis Sci 42:305-312.

Krishna VR, Alexander KR, Peachey NS (2002) Temporal properties of the mouse cone electroretinogram. J Neurophysiol 87:42-48.

Krispel CM, Chen CK, Simon MI, Burns ME (2003) Novel form of adaptation in mouse retinal rods speeds recovery of phototransduction. J Gen Physiol 122:703-712.

Krispel CM, Chen D, Melling N, Chen YJ, Martemyanov KA, Quillinan N, Arshavsky VY, Wensel TG, Chen CK, Burns ME (2006) RGS expression rate-limits recovery of rod photoresponses. Neuron 51:409-416.

Lall GS, Revell VL, Momiji H, Al Enezi J, Altimus CM, Güler AD, Aguilar C, Cameron MA, Allender S, Hankins MW, Lucas RJ (2010) Distinct contributions of rod, cone, and melanopsin photoreceptors to encoding irradiance. Neuron 66:417-428.

Lamb TD (2013) Evolution of phototransduction, vertebrate photoreceptors and retina. Prog Retin Eye Res 36:52-119.

Lobanova ES, Finkelstein S, Song H, Tsang SH, Chen CK, Sokolov M, Skiba NP, Arshavsky VY (2007) Transducin translocation in rods is triggered by saturation of the GTPase-activating complex. J Neurosci 27:11511160.

Lyubarsky AL, Daniele LL, Pugh EN Jr (2004) From candelas to photoisomerizations in the mouse eye by rhodopsin bleaching in situ and the light-rearing dependence of the major components of the mouse ERG. Vision Res 44:3235-3251.

MacLeod DI (1972) Rods cancel cones in flicker. Nature 235:173-174.

Macmillan N, Creelman C (2005) Detection theory: a user's guide. New York: Lawrence Erlbaum.

Matthews HR, Murphy RL, Fain GL, Lamb TD (1988) Photoreceptor light adaptation is mediated by cytoplasmic calcium concentration. Nature 334:67-69.

Millar RB (2011) Maximum likelihood estimation and inference. Chichester, West Sussex, UK: Wiley.

Naarendorp F, Sato Y, Cajdric A, Hubbard NP (2001) Absolute and relative sensitivity of the scotopic system of rat: electroretinography and behavior. Vis Neurosci 18:641-656.

Naarendorp F, Esdaille TM, Banden SM, Andrews-Labenski J, Gross OP, Pugh EN Jr (2010) Dark light, rod saturation, and the absolute and incremental sensitivity of mouse cone vision. J Neurosci 30:12495-12507.

Nathan J, Reh R, Ankoudinova I, Ankoudinova G, Chang B, Heckenlively J, Hurley JB (2006) Scotopic and photopic visual thresholds and spatial and temporal discrimination evaluated by behavior of mice in a water maze. Photochem Photobiol 82:1489-1494.

Nikonov S, Lamb TD, Pugh EN Jr (2000) The role of steady phosphodiesterase activity in the kinetics and sensitivity of the light-adapted salamander rod photoresponse. J Gen Physiol 116:795-824.

Nusinowitz S, Ridder WH 3rd, Ramirez J (2007) Temporal response properties of the primary and secondary rod-signaling pathways in normal and Gnat2 mutant mice. Exp Eye Res 84:1104-1114.

Oesch NW, Diamond JS (2011) Ribbon synapses compute temporal contrast and encode luminance in retinal rod bipolar cells. Nat Neurosci 14:1555-1561.

Okawa H, Miyagishima KJ, Arman AC, Hurley JB, Field GD, Sampath AP (2010) Optimal processing of photoreceptor signals is required to maximize behavioural sensitivity. J Physiol 588:1947-1960.

Oppenheim AV, Willsky AS, Young IT (1983) Signals and systems. London: Prentice Hall.
Parker AJ, Newsome WT (1998) Sense and the single neuron: probing the physiology of perception. Ann Rev Neurosci 21:227-277.

Peinado Allina G, Fortenbach C, Naarendorp F, Gross OP, Pugh EN Jr, Burns ME (2017) Bright flash response recovery of mammalian rods in vivo is rate limited by RGS9. J Gen Physiol 149:443-454.

Pelli DG, Farell B (1999) Why use noise? J Opt Soc Am A Opt Image Sci Vis $16: 647-653$.

Purpura K, Tranchina D, Kaplan E, Shapley RM (1990) Light adaptation in the primate retina: analysis of changes in gain and dynamics of monkey retinal ganglion cells. Vis Neurosci 4:75-93.

Rieke F (2001) Temporal contrast adaptation in salamander bipolar cells. J Neurosci 21:9445-9454

Rivlin-Etzion M, Grimes WN, Rieke F (2018) Flexible neural hardware supports dynamic computations in retina. Trends Neurosci 41:224-237.

Robson JG, Frishman LJ (1995) Response linearity and kinetics of the cat retina: the bipolar cell component of the dark-adapted electroretinogram. Vis Neurosci 12:837-850.

Robson JG, Frishman LJ (2014) The rod-driven a-wave of the dark-adapted mammalian electroretinogram. Prog Retin Eye Res 39:1-22.

Rovamo J, Raninen A, Donner K (1999) The effects of temporal noise and retinal illuminance on foveal flicker sensitivity. Vision Res 39:533-550.

Sampath AP, Strissel KJ, Elias R, Arshavsky VY, McGinnis JF, Chen J, Kawamura S, Rieke F, Hurley JB (2005) Recoverin improves rod-mediated vision by enhancing signal transmission in the mouse retina. Neuron 46:413-420.

Saszik SM, Robson JG, Frishman LJ (2002) The scotopic threshold response of the dark-adapted electroretinogram of the mouse. J Physiol 543:899916.

Seiple W, Holopigian K, Greenstein V, Hood DC (1992) Temporal frequency dependent adaptation at the level of the outer retina in humans. Vision Res 32:2043-2048.

Shapley R, Enroth-Cugell C (1984) Visual adaptation and retinal gain controls. Prog Retin Res 3:263-364.

Sharpe LT, Stockman A (1999) Rod pathways: the importance of seeing nothing. Trends Neurosci 22:497-504.

Sharpe LT, Stockman A, MacLeod DI (1989) Rod flicker perception: scotopic duality, phase lags and destructive interference. Vision Res 29:1539_ 1559.

Shirato S, Maeda H, Miura G, Frishman LJ (2008) Postreceptoral contributions to the light-adapted ERG of mice lacking b-waves. Exp Eye Res 86:914-928.

Sinha R, Hoon M, Baudin J, Okawa H, Wong ROL, Rieke F (2017) Cellular and circuit mechanisms shaping the perceptual properties of the primate fovea. Cell 168:413-426.e412.

Smith VC, Pokorny J, Lee BB, Dacey DM (2001) Primate horizontal cell dynamics: an analysis of sensitivity regulation in the outer retina. J Neurophysiol 85:545-558.

Smith VC, Pokorny J, Lee BB, Dacey DM (2008) Sequential processing in vision: the interaction of sensitivity regulation and temporal dynamics. Vision Res 48:2649-2656.

Sokolov M, Lyubarsky AL, Strissel KJ, Savchenko AB, Govardovskii VI, Pugh EN Jr, Arshavsky VY (2002) Massive light-driven translocation of transducin between the two major compartments of rod cells: a novel mechanism of light adaptation. Neuron 34:95-106.

Stockman A, Sharpe LT (2006) Into the twilight zone: the complexities of mesopic vision and luminous efficiency. Ophthalmic Physiol Opt 26:225239.

Stockman A, Sharpe LT, Zrenner E, Nordby K (1991) Slow and fast pathways in the human rod visual system: electrophysiology and psychophysics. J Opt Soc Am A 8:1657-1665.

Stockman A, Langendörfer M, Smithson HE, Sharpe LT (2006) Human cone light adaptation: from behavioral measurements to molecular mechanisms. J Vis 6(11):5 1194-1213.

Stockman A, Candler T, Sharpe LT (2010) Human scotopic sensitivity is regulated postreceptorally by changing the speed of the scotopic response. J Vis 10(2):12 1-19.

Tranchina D, Gordon J, Shapley RM (1984) Retinal light adaptation: evidence for a feedback mechanism. Nature 310:314-316.

Tranchina D, Sneyd J, Cadenas ID (1991) Light adaptation in turtle cones: testing and analysis of a model for phototransduction. Biophys J 60:217237.

Tyler CW (1985) Analysis of visual modulation sensitivity. II. Peripheral 
retina and the role of photoreceptor dimensions. J Opt Soc Am A 2:393-398.

Umino Y, Herrmann R, Chen CK, Barlow RB, Arshavsky VY, Solessio E (2012) The relationship between slow photoresponse recovery rate and temporal resolution of vision. J Neurosci 32:14364-14373.

Umino Y, Pasquale R, Solessio E (2018) Visual temporal contrast sensitivity in the behaving mouse shares fundamental properties with human psychophysics. eNeuro 5:ENEURO.0181-18.2018.

van den Berg TJ, Spekreijse H (1977) Interaction between rod and cone signals studied with temporal sine wave stimulation. J Opt Soc Am 67:1210-1217.

Vinberg F, Kolesnikov AV, Kefalov VJ (2014) Ex vivo ERG analysis of photoreceptors using an in vivo ERG system. Vision Res 101:108-117.

Völgyi B, Deans MR, Paul DL, Bloomfield SA (2004) Convergence and segregation of the multiple rod pathways in mammalian retina. J Neurosci 24:11182-11192.
Walraven J, Enroth-Cugell C, Hood DC, MacLeod DI, Schnapf JL (1990) The control of visual sensitivity: receptoral and postreceptoral processes. In: Visual perception: the neurophysiological foundations (Spillmann L, Werner JS, eds). San Diego: Academic.

Wang YV, Weick M, Demb JB (2011) Spectral and temporal sensitivity of cone-mediated responses in mouse retinal ganglion cells. J Neurosci 31:7670-7681.

Watson A (1986) Temporal sensitivity. In: Handbook of perception and human performance (Boff K, Kaufman L, Thomas J, eds). New York: Wiley.

Wyszecki G, Stiles WS (2000) Color science: concepts and methods, quantitative data and formulae. New York: Wiley.

Yau KW, Nakatani K (1985) Light-induced reduction of cytoplasmic free calcium in retinal rod outer segment. Nature 313:579-582.

Zele AJ, Cao D (2014) Vision under mesopic and scotopic illumination. Front Psychol 5:1594. 